\title{
Contribution of the Clp Protease to Bacterial Survival and Mitochondrial Homoeostasis
}

\author{
Astrid Illigmann ${ }^{a}$ Yvonne Thoma $^{\mathrm{a}}$ Stefan Pan ${ }^{\mathrm{a}}$ Laura Reinhardt ${ }^{\mathrm{a}}$ \\ Heike Brötz-Oesterhelt ${ }^{a, b}$ \\ aDepartment of Microbial Bioactive Compounds, Interfaculty Institute of Microbiology and Infection \\ Medicine, University of Tübingen, Tübingen, Germany; ${ }^{b}$ Cluster of Excellence Controlling Microbes to \\ Fight Infection, University of Tübingen, Tübingen, Germany
}

\section{Keywords}

ClpP $\cdot$ Clp-ATPase $\cdot$ Stress response $\cdot$ Regulatory proteolysis $\cdot$ Pathogenesis

\begin{abstract}
Fast adaptation to environmental changes ensures bacterial survival, and proteolysis represents a key cellular process in adaptation. The Clp protease system is a multi-component machinery responsible for protein homoeostasis, protein quality control, and targeted proteolysis of transcriptional regulators in prokaryotic cells and prokaryote-derived organelles of eukaryotic cells. A functional Clp protease complex consists of the tetradecameric proteolytic core CIpP and a hexameric ATP-consuming Clp-ATPase, several of which can associate with the same proteolytic core. Clp-ATPases confer substrate specificity by recognising specific degradation tags, and further selectivity is conferred by adaptor proteins, together allowing for a fine-tuned degradation process embedded in elaborate regulatory networks. This review focuses on the contribution of the Clp protease system to prokaryotic survival and summarises the current state of knowledge for exemplary bacteria in an increasing degree of
\end{abstract}

interaction with eukaryotic cells. Starting from free-living bacteria as exemplified by a non-pathogenic and a pathogenic member of the Firmicutes, i.e., Bacillus subtilis and Staphylococcus aureus, respectively, we turn our attention to facultative and obligate intracellular bacterial pathogens, i.e., Mycobacterium tuberculosis, Listeria monocytogenes, and Chlamydia trachomatis, and conclude with mitochondria. Under stress conditions, the Clp protease system exerts its pivotal role in the degradation of damaged proteins and controls the timing and extent of the heat-shock response by regulatory proteolysis. Key regulators of developmental programmes like natural competence, motility, and sporulation are also under Clp proteolytic control. In many pathogenic species, the Clp system is required for the expression of virulence factors and essential for colonising the host. In accordance with its evolutionary origin, the human mitochondrial Clp protease strongly resembles its bacterial counterparts, taking a central role in protein quality control and homoeostasis, energy metabolism, and apoptosis in eukaryotic cells, and several cancer cell types depend on it for proliferation.

(c) 2021 The Author(s)

Published by S. Karger AG, Basel
(C) 2021 The Author(s)

Published by S. Karger AG, Basel

This article is licensed under the Creative Commons Attribution 4.0 International License (CC BY) (http://www.karger.com/Services/ OpenAccessLicense). Usage, derivative works and distribution are permitted provided that proper credit is given to the author and the original publisher.
Correspondence to:

Heike Brötz-Oesterhelt, heike.broetz-oesterhelt@ uni-tuebingen.de 


\section{Introduction to the Clp Machinery}

The caseinolytic protease Clp, an energy-dependent, multimeric degradation machinery, plays a crucial role in protein homoeostasis and quality control in prokaryotic cells and prokaryote-derived organelles of eukaryotic cells. The finely tuned Clp system is pivotal for bacterial survival under stress conditions, as damaged proteins are recognised as substrates [Meyer and Baker, 2011] and a multitude of major stress regulators are controlled by Clp-mediated regulatory proteolysis in various organisms [Frees et al., 2007]. Heat-shock chaperone induction, natural competence, motility, and sporulation are escape strategies regulated by the Clp machinery [Frees et al., 2007; Elsholz et al., 2017], and many pathogenic species were attenuated in the host environment when components of the Clp protease system were deleted [BrötzOesterhelt and Sass, 2014].

A functional Clp protease complex is composed of an ATP-consuming hexameric Clp-ATPase, which belongs to the $\mathrm{Clp} / \mathrm{Hsp} 100$ chaperones of the AAA+ (ATPases associated with cellular activities) superfamily and the barrel-shaped proteolytic core ClpP (Fig. 1a, b). The central component $\mathrm{ClpP}$ is a self-compartmentalised serine peptidase with catalytic triads secluded within an internal degradation chamber formed by two stacked heptameric rings. The ClpP peptidase itself exhibits solely peptidolytic activity, whereas for protein hydrolysis it associates with Clp-ATPases, which unfold and translocate protein substrates in an ATP-consuming process into the secluded degradation chamber of ClpP [Olivares et al., 2016]. The catalytic triads of ClpP have very low cutting preference, and substrate specificity is conferred by the cognate Clp-ATPases. Usually, bacteria possess different ClpATPases, such as ClpX, ClpA, ClpC, or ClpE, which interact with the same proteolytic core [Frees et al., 2007; Olivares et al., 2016]. In this review, we use the term " $\mathrm{Clp}$ protease" to include all Clp-ATPase/ClpP combinations of a given organism to signify that they share the same proteolytic core.

Clp-ATPases recognise their substrates via certain degradation tags, which are either intrinsic to a particular protein sequence and only exposed under certain conditions [Maglica et al., 2008], or attached to proteins on demand, such as the ssrA-tag translated from a transfermessenger (tm)RNA to rescue stalled ribosomes [Flynn et al., 2001]. Substrate specificity is further regulated by the interaction of the Clp-ATPases with specific adaptor proteins, like SspB and ClpS in Escherichia coli or McsB in Bacillus subtilis [Kirstein et al., 2009b]. Adaptor pro-

Clp Protease in Homoeostasis and Survival teins can, for example, enhance the affinity of a given ClpATPase to a particular protein substrate or stabilise the functional oligomeric state of a Clp-ATPase [Kirstein et al., 2009b]. Thus, the adaptor proteins offer an additional level of fine regulation to modulate Clp protease activity. For instance, in E. coli, ssrA-tagged proteins can be degraded by ClpXP as well as ClpAP, and the two adaptors SspB and ClpS help direct the substrate flow between the two protease complexes [Kirstein et al., 2009b]. Phosphorylation is a further means of regulating proteolysis. In B. subtilis, the arginine kinase McsB not only acts as an adaptor protein but distinguishes proteins via arginine phosphorylation for better recognition by $\mathrm{ClpC}$ and subsequent degradation by ClpCP [Trentini et al., 2016].

Most bacterial species encode only a single $c l p P$ gene, resulting in a homo-tetradecameric proteolytic core with 14 identical subunits. However, the increasing number of studies on ClpP proteins from pathogenic bacteria such as Chlamydia trachomatis, Clostridium difficile, Mycobacterium tuberculosis, Listeria monocytogenes, or Pseudomonas aeruginosa revealed the existence of distinct $\mathrm{ClpP}$ isoforms within the same organism, forming distinct homomeric tetradecamers or mixed heteromeric tetradecamers [Schmitz et al., 2014; Hall et al., 2017; Gatsogiannis et al., 2019; Lavey et al., 2019; Pan et al., 2019]. For instance, the intracellular pathogens $M$. tuberculosis, L. monocytogenes, and C. trachomatis contain a mixed ClpP1P2 hetero-tetradecamer, one heptameric ring comprising only ClpP1 and the opposite ring only ClpP2 [Schmitz et al., 2014; Gatsogiannis et al., 2019; Pan et al., 2019], as shown in Figure 1c.

This review starts with a free-living non-pathogenic soil organism to continue with pathogens in increasing dependence on host cells and concludes with mitochondria as an example for a bacteria-derived organelle. In following this structure, it also starts with Clp systems studied for many years, where a substantial level of information exists, regarding structure, regulatory networks, and physiological targets, towards systems studied only more recently and where few, if any, natural substrates are known.

\section{B. subtilis: Role of Clp in Protein Homoeostasis and Environmental Stress Management}

The Clp machinery of $B$. subtilis was among the first studied, with a particular interest in regulatory proteolysis networks. Here, B. subtilis is discussed as an example of the contribution of the Clp protease to a versatile bac- 


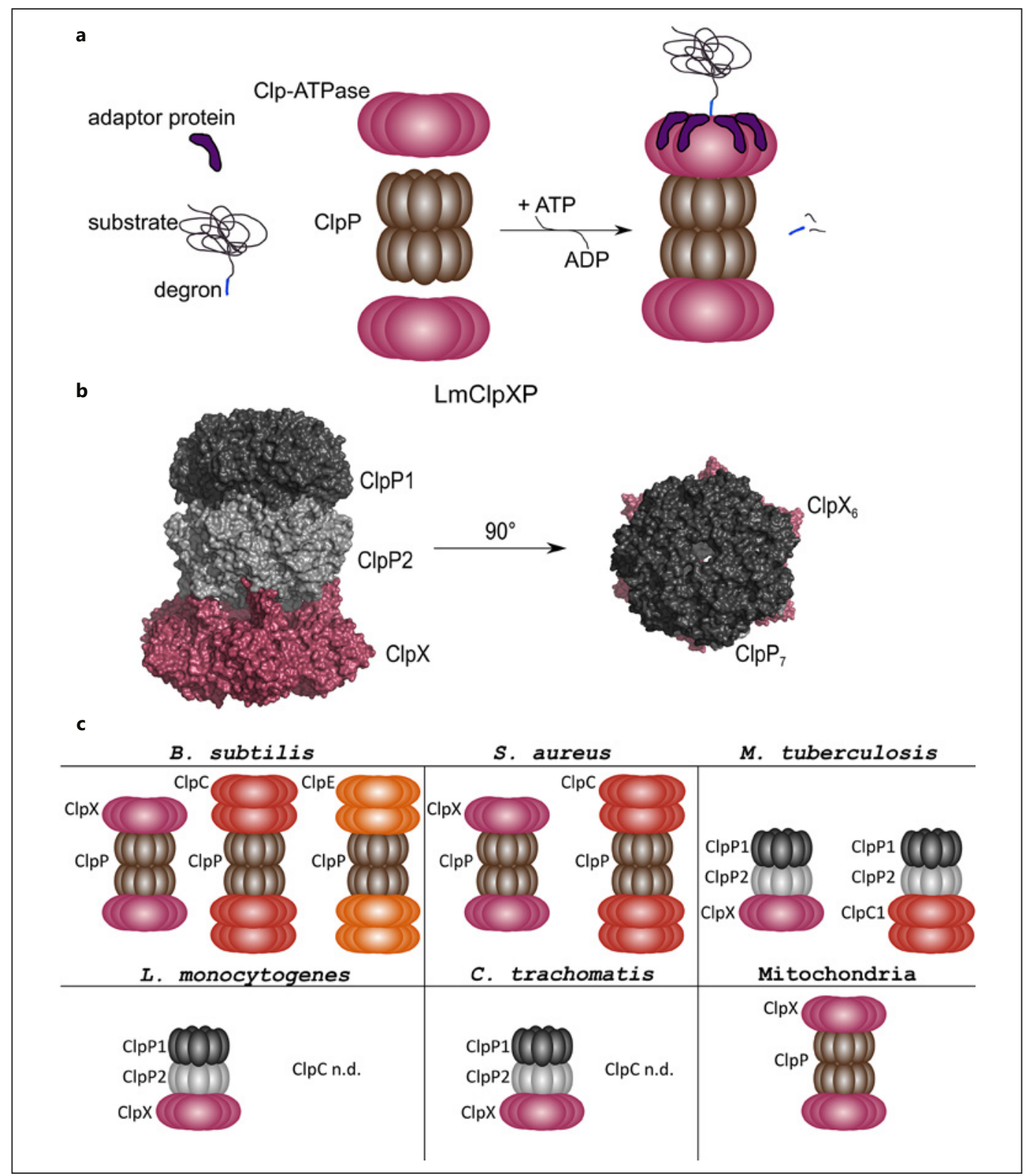

Fig. 1. Operation mode and composition of the exemplary Clp protease complexes discussed in this review. a Model for the general operation mode of a Clp protease complex. Clp-ATPase-mediated substrate degradation by the proteolytic core ClpP is fuelled by ATP hydrolysis to unfold and translocate the polypeptide chain into the degradation chamber of ClpP. Further substrate specificity is achieved by the interaction of the Clp-ATPases with specific adaptors. b Cryo-EM structure of the L. monocytogenes ClpX hexamer (PDB: 6SFW) in interaction with the heptameric binding surface of the exclusive interaction partner ClpP2 within the hetero-tetradecameric Listeria ClpP1P2 (PDB: 6SFX) proteolytic core [Gatsogiannis et al., 2019]. The Listeria ClpXP1P2 cryo-EM structure is depicted in a surface fill model representation with ClpX coloured in raspberry red and ClpP1 and ClpP2 in dark and light grey, respectively. A $90^{\circ}$ rotation of the cryo-EM structure, now exposing the apical surface of $\mathrm{ClpP} 1$, illustrates the symmetry mis- match between the hexameric ClpX unfoldase and the heptameric ClpP1P2 core. The structures were visualised using PYMOL (https://pymol.org/2/). c Schematic overview of the composition of selected Clp protease complexes. In principle, due to the homomeric nature of the proteolytic ClpP core in B. subtilis and S. aureus, the Clp-ATPases ClpX, ClpC, and in B. subtilis also ClpE can bind to both sides of the tetradecameric barrel, which also refers to mitochondrial ClpX and ClpP [Kang et al., 2002]. Contrariwise, in L. monocytogenes, $M$. tuberculosis, and C. trachomatis, comprising a heteromeric ClpP1P2 core, ClpP2 acts as the exclusive interaction partner for the Clp-ATPases ClpX and ClpC1 in M. tuberculosis [Leodolter et al., 2015], ClpX in L. monocytogenes [Gatsogiannis et al., 2019], and ClpX in C. trachomatis [Pan et al., 2019]. The composition of ClpCP in L. monocytogenes and C. trachomatis has not been determined (n.d.) so far. 
terial lifestyle and efficient stress compensation. B. subtilis masters survival in diverse environments, such as soil, marine ecosystems, the gut of invertebrates and vertebrates, and in association with plant roots [Brito et al., 2018]. Adaptation to different environments and changes within a given environment requires flexible adjustment of the bacterial proteome. Besides differential protein expression and activation, targeted proteolysis of regulatory factors plays a crucial role in managing the required alterations, as does the degradation of misfolded or aggregated proteins accumulating under stress. AAA+ proteases carry out this task, and the Clp protease is a prominent member of those [Kirstein et al., 2009b].

Prokaryotic organisms are directly affected by changes in their environment, and adverse conditions, such as nutrient shortage, unfavourable temperature, or exposure to toxic agents, require an immediate protective response and sometimes the induction of a long-term survival programme. In B. subtilis, regulatory proteolysis by the Clp machinery helps to: (1) better cope with external stress such as elevated temperatures, (2) actively move to a more favourable environment, (3) take up external DNA from the environment to enrich the gene pool, and, should everything else fail, to (4) secure long-term survival by starting the sporulation programme (Fig. 2a).

In B. subtilis, ClpP, as well as the ATPases ClpC and ClpE, belong to the heat-shock regulon. $C l p P$ is transcribed as a monocistronic gene, $c l p C$ forms an operon with the adaptors $m c s A$ and $m c s B$, while $\operatorname{clp} X$ and $c l p E$ are encoded in distinct operons [Derre et al., 1999; Krüger et al., 2001; Frees et al., 2007]. To allow for well-restrained proteolysis in general and efficient but transient induction under stress, the Clp system is controlled by several regulatory circles. Interestingly, the Clp machinery autoregulates its expression by recognising its own repressor CtsR as a proteolytic substrate. CtsR is a dimeric repressor, highly conserved in low-GC Gram-positive bacteria and positioned as the first gene in the $c l p C$ operon. The repressor negatively regulates the expression of $c l p P, c l p E$, and the $c l p C$ operon (including its own gene) by binding to a hepta-nucleotide direct repeat within the respective promoter regions and interfering with the RNA polymerase E6A holoenzyme [Derre et al., 1999; Krüger et al., 2001; Elsholz et al., 2010b]. Under non-stressing conditions, the active conformation of CtsR binds to DNA, repressing the transcription of $c l p P, c l p E$, and $c l p C$ and its own expression. Upon heat exposure, CtsR acts as a thermosensor and undergoes a conformational change that reduces its binding affinity for DNA [Derre et al., 2000; Elsholz et al., 2010b]. CtsR-controlled genes are tran-

Clp Protease in Homoeostasis and Survival scribed, i.e., the respective $c l p$ genes and many other heatshock genes. McsA and McsB play a central role in CtsR degradation during heat stress, and auto-phosphorylation of McsB is essential for CtsR degradation by ClpCP [Kirstein et al., 2005; Kirstein et al., 2007; Elsholz et al., 2010b]. After initial strong heat-shock protein expression, the heat-shock response declines. CtsR is re-activated after $30 \mathrm{~min}$ of heat exposure, and phosphorylated McsB is either de-phosphorylated by YwlE or rapidly degraded by ClpCP to prevent the degradation of activated CtsR [Elsholz et al., 2010b]. For an extensive overview outlining the regulation of CtsR we refer readers to Frees et al. [2007] and Elsholz et al. [2010a]. Besides ClpCP, ClpEP and ClpXP were also shown to degrade CtsR, albeit under different conditions. While ClpEP degrades CtsR upon severe heat-shock, ClpXP degrades CtsR at $37^{\circ} \mathrm{C}$ [Derre et al., 2000; Krüger et al., 2001; Miethke et al., 2006]. ClpC, independently of ClpP, is presumed to play a positive role by binding and protecting CtsR against degradation by $\mathrm{ClpXP}$ at $37^{\circ} \mathrm{C}$ [Derre et al., 2000]. ClpX does not belong to the CtsR regulon, and its accumulation during heat stress is prevented due to instability of the $c l p X$ mRNA at $50^{\circ} \mathrm{C}$ [Gerth et al., 1996; Gerth et al., 2004].

A further level of regulation is added to the heat-shock response by a regulatory feedback loop between the transcription factor Spx and the CtsR regulon. Spx is well known as an oxidative stress regulator, and in addition Spx plays an important role in the heat-shock response and thermotolerance development [Runde et al., 2014]. Spx was shown to induce the CtsR regulon and directly mediate $y j b H$ and $c l p X$ expression [Rochat et al., 2012]. $\mathrm{ClpXP}$, together with the adaptor YjbH, degrades Spx under non-stressed conditions [Garg et al., 2009; Nakano et al., 2002b; Larsson et al., 2007; Runde et al., 2014], and the induction of $c l p X$ and $y j b H$ expression by Spx was discussed to represent a negative regulatory loop, promoting the reduction of Spx levels [Rochat et al., 2012]. However, upon oxidative stress, ClpXP activity is impaired [Zhang and Zuber, 2007], and when ClpXP is incapable of degrading Spx, ClpCP controls the cellular Spx levels [Rojas-Tapias and Helmann, 2019]. Moreover, McsB modulates Spx activity through arginine phosphorylation and promotes Spx degradation by ClpCP as an adaptor protein [Rojas-Tapias and Helmann, 2019]. In short, the transcriptional regulator Spx positively contributes to the activity of the CtsR regulon, and induces also the expression of $c l p X$ and $y j b H$, whereas the CtsR regulon and especially the Clp proteases together with their adaptors mediate the inactivation of Spx, preventing negative effects of Spx overaccumulation, i.e., reduced growth, spor- 


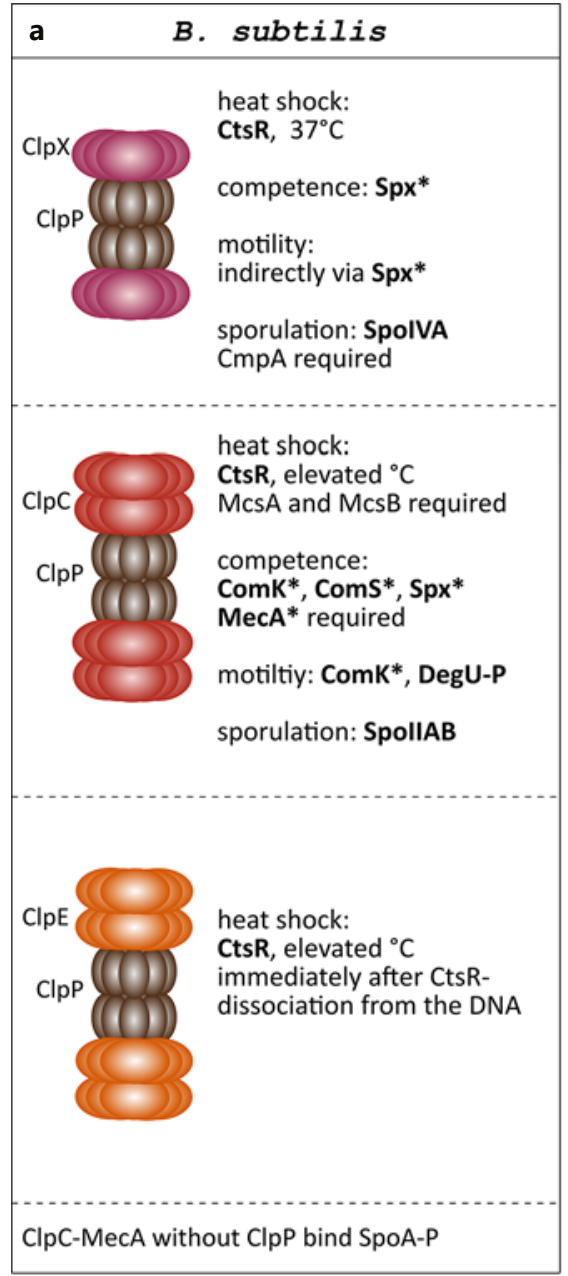

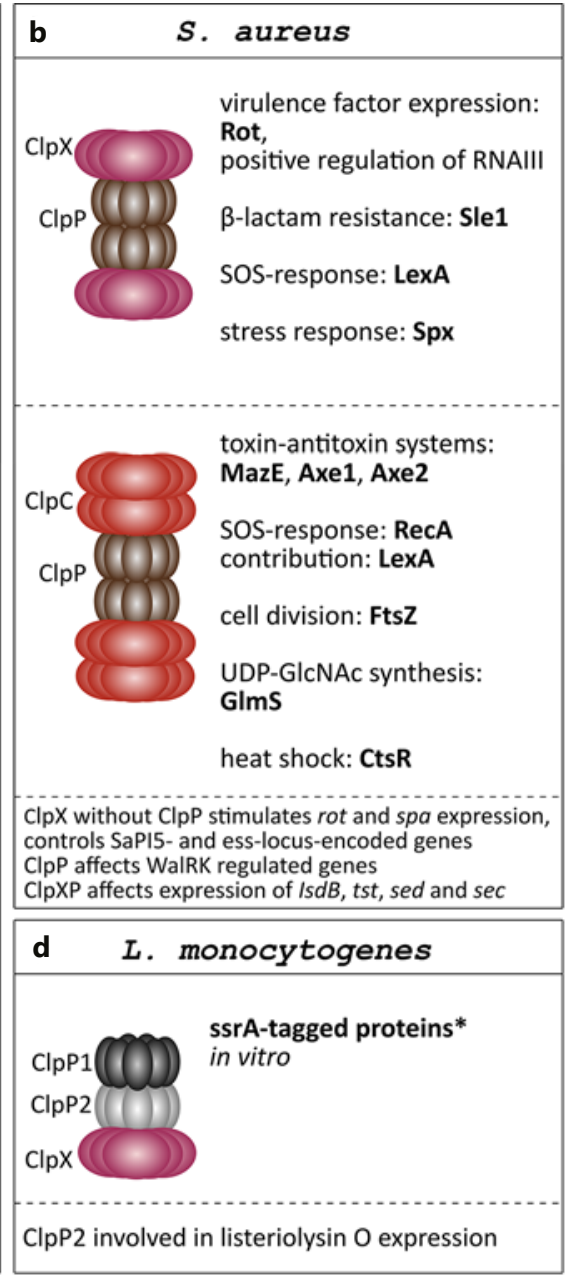

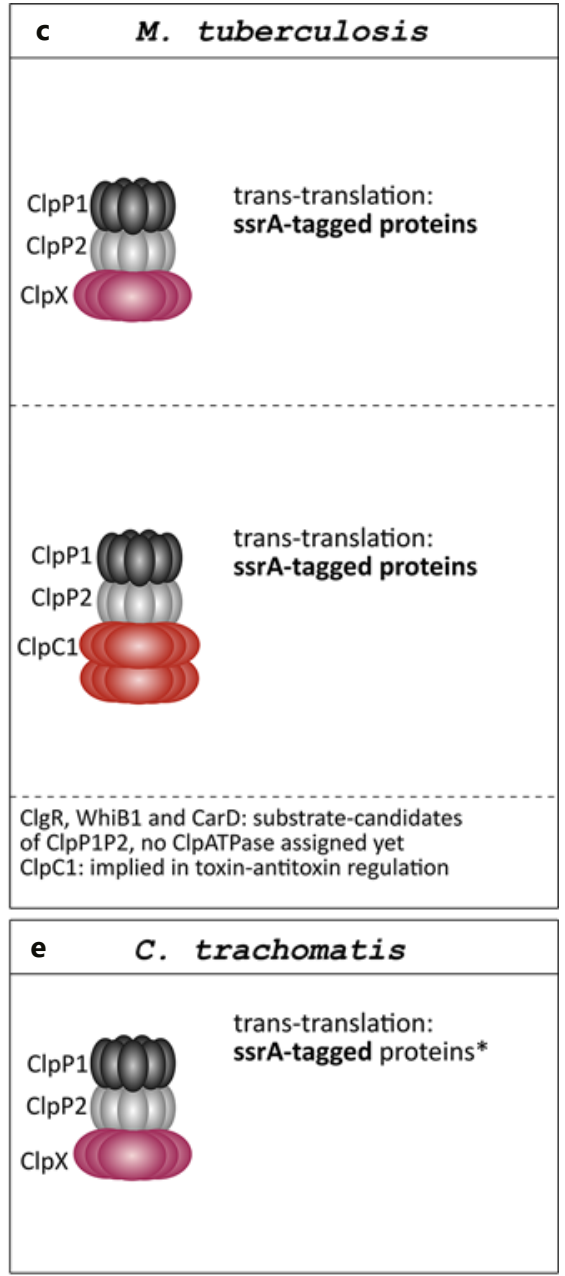

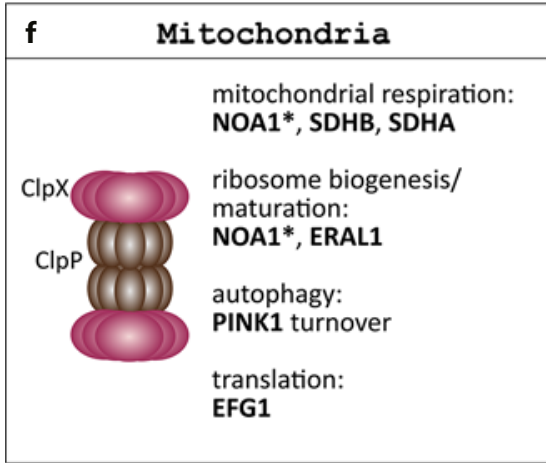

Fig. 2. Natural substrates of Clp proteases and physiological roles discussed in this review. Clp proteases of B. subtilis (a), S. aureus (b), M. tuberculosis (c), L. monocytogenes (d), C. trachomatis (e), and mitochondria (f), and the functions reported so far. In all the heteromeric ClpP1P2 complexes discussed in this review, ClpATPase interaction was only observed with ClpP2. Exemplary natural protein targets discussed in this review and shown to accumulate in $c l p$ deletion mutants and/or to interact in Clp pull-down assays and/or supported by physiological evidence in whole-cell studies are marked in bold. Substrates for which we are aware of in vitro digestion are marked by an asterisk [Turgay, 1998; Nakano et al., 2002b; Nakano et al., 2003; Kirstein et al., 2009a; Al-Furoukh et al., 2014; Balogh et al., 2017; Pan et al., 2019]. In addition, most Clp proteases are also involved in general protein quality control. For an extensive overview of Clp protease substrates, we refer readers to Feng et al. [2013] and Bhandari et al. [2018]. 
ulation, and competence as observed in $\Delta y j b H, \Delta c l p X$, and $\Delta c l p C$ mutants [Nakano et al., 2002b; Larsson et al., 2007]. This well-studied example of the heat-shock response illustrates how multilayered circles of regulatory proteolysis can rapidly elicit and later quench a stress response.

A disbalance in protein homoeostasis can result from external hazards such as heat stress or exposure to antibacterial agents, and ClpP, ClpC, ClpX, and ClpE help B. subtilis maintain a balanced, functional proteome in a changing environment. Their adhesion to aggregated heat-damaged proteins, as well as their degradation of defective proteins, clearly demonstrate their role in protein quality control, especially under stress conditions [Krüger et al., 2000; Miethke et al., 2006]. Additionally, ClpP and $\mathrm{ClpC}$ participate in general protein turnover under nonstressed conditions, again efficiently preventing the accumulation of protein aggregates [Krüger et al., 2000; Kock et al., 2004]. Proteome rearrangements are also important during shifts of growth phases and nutrient shortage. Although $c l p P$ is dispensable for $B$. subtilis during exponential growth in rich media at moderate temperatures, the Clp protease becomes essential when cells enter stationary phase or are grown in minimal media [Msadek et al., 1998]. Typical stationary phase adaptive responses, such as degradative enzyme synthesis, motility, natural genetic competence, and sporulation, are regulated by ClpP [Msadek et al., 1998]. In the light of survival strategies, motile cells have the advantage that they can actively change their environment by moving from unfavourable to more favourable conditions. In that sense, flagella represent an efficient coping strategy. Flagella are elaborate proton motive force-fuelled, rotating machines, and their assembly depends on the coordinated expression of various genes and transcription factors, among them DegU and $6^{\mathrm{D}}$ [Tsukahara and Ogura, 2008]. The alternative sigma factor $6^{\mathrm{D}}$ is encoded on the fla/che operon besides other flagella machinery genes. Once $6^{\mathrm{D}}$ is activated, genes required for filament synthesis are expressed, including hag [Mukherjee and Kearns, 2014]. The Hag protein represents the flagellin monomer, and about 12,000 subunits form one flagellum [Oshiro et al., 2019]. In $B$. subtilis, deletion of $c l p P, c l p C, c l p X$, or the adaptor mec $A$ results in the loss of motility [Liu and Zuber, 1998; Msadek et al., 1998; Molière et al., 2016]. One way for ClpCP to regulate the swimming motility in B. subtilis is via ComK. The ComK transcription factor, which also plays a major role in competence regulation (see below), indirectly promotes the expression of $f l g M$, which encodes the anti-sigma factor of $6^{\mathrm{D}}$. ComK is a ClpCP substrate, and by de-

Clp Protease in Homoeostasis and Survival grading ComK, $\mathrm{ClpCP}$ positively affects $6^{\mathrm{D}}$-dependent hag transcription, followed by flagellar assembly [Liu and Zuber, 1998]. Moreover, ClpCP targets DegU in its phosphorylated form [Molière et al., 2016]. DegU is the response regulator of the DegS-DegU two-component system that controls several post-exponential responses, among them motility. High DegU-P levels prevent expression of the fla/che operon, while DegU activates the same operon in its unphosphorylated or low-phosphorylated state. In contrast to the direct effects of ClpCP, ClpXP stimulates motility indirectly through degradation of the oxidative stress regulator Spx, a repressor of the fla/che operon and hag expression [Molière et al., 2016].

Developmental processes during stationary phase also include the physiological state of natural competence. Natural competence allows a subpopulation of B. subtilis cells to import foreign DNA, thereby enriching the gene pool [Turgay et al., 1997]. The autoregulatory transcription factor ComK is also central to competence development [Liu and Zuber, 1998]. During exponential growth, the adaptor MecA targets ComK for degradation by ClpCP, preventing its accumulation [Turgay et al., 1997; Turgay, 1998]. However, dependent on quorum-sensing mediated signals, the response regulator ComA is phosphorylated. ComA-P interacts with the RNA polymerase (RNAP), activating the transcription of the srf operon, resulting in the synthesis of the anti-adaptor protein ComS [D'Souza et al., 1994; Hamoen et al., 1995]. ComS binds to MecA, thus releasing ComK to initiate competence gene transcription. Thereby, ComS and MecA become substrates for ClpCP-mediated degradation themselves [Turgay et al., 1997, 1998]. In the context of natural competence, the transcription factor Spx prevents the development of this physiological state. Spx forms a quaternary complex with ComK-MecA-ClpC, resulting in enhanced binding of ComK to MecA-ClpC and inhibition of competence development [Nakano et al., 2002a]. Notably, Spx also represents a ClpCP-MecA substrate, clearly shown to be degraded in vitro [Nakano et al., 2002b] as well as in vivo [Rojas-Tapias and Helmann, 2019]. The fact that Spx and ComK are both substrates of the same Clp protease complex involving the same adaptor, and even being bound simultaneously, suggests competition of both substrates during the degradation process by Clp$\mathrm{CP}-\mathrm{MecA}$. Furthermore, Spx binds to the $\alpha$-subunit of RNAP, preventing competence development by disrupting ComA-P-RNAP mediated transcription initiation [Nakano et al., 2003; Zhang et al., 2006]. Eventually, ComS is not synthesised, leading to the degradation of ComK and inhibition of competence. ClpXP contributes 
to $s r f$ transcription activation, which results in ComS synthesis, by degradation of Spx [Nakano et al., 2000; Nakano et al., 2003]. Hence, the lack of competence in $c l p P$ and $c l p X$ deletion mutants results from an accumulation of Spx [Nakano et al., 2002b].

Genes controlling competence and sporulation are organised in tightly interconnected networks [Grossman, 1995]. Therefore, it is reasonable that the Clp protease is involved in both physiological processes. For instance, mutants deficient in ClpP show a defect in both competence development and sporulation, and mutants lacking $\mathrm{ClpC}$ are deficient in sporulation, as well [Msadek et al., 1998]. A rationale why $\mathrm{ClpC}$ and $\mathrm{ClpP}$ positively regulate sporulation was provided by the observation that SpoIIAB is specifically degraded by ClpCP [Pan et al., 2001]. SpoIIAB is an anti-sigma factor, sequestering $6^{\mathrm{F}}$, a forespore-specific sigma factor, in an inactive state. However, expression of $6^{\mathrm{F}}$ and other sigma factors essential for sporulation depends also on the accumulation of Spo0A, a transcription factor and the master regulator of sporulation, in its phosphorylated form [Grossman, 1995]. In this regard, it was recently found that Spo0A-P binds to $\mathrm{ClpC}-\mathrm{MecA}$, independently of ClpP. Binding of Spo0A-P by $\mathrm{ClpC}-\mathrm{MecA}$ might result in a bulky complex and prevent recruitment of the RNA polymerase. This way, ClpC-MecA directly repress the transcription of Spo0Adependent genes without degrading the actual target [Tanner et al., 2018]. These examples show how a ClpATPase can have opposing roles in the regulation of the same developmental process, thereby allowing for finetuning. While $\mathrm{ClpCP}$ and $\mathrm{ClpC}-\mathrm{MecA}$ affect sporulation at an early stage, ClpXP plays an important role at a later stage during sporulation, i.e., quality control of the spore envelope. SpoIVA is essential for the formation of the spore basement layer, on top of which coat proteins assemble [Roels et al., 1992; Mckenney et al., 2013]. In cells defective in spore envelope assembly, CmpA mediates degradation of SpoIVA by ClpXP, eventually leading to the destabilisation of the forespore and resulting in cell lysis. By participating in a critical checkpoint of the sporulation program, ClpXP and CmpA preserve the integrity of the bacterial population [Tan et al., 2015].

In summary, the Clp system in B. subtilis coordinates a variety of tasks, ranging from fundamental stress responses to regulatory proteolysis of specific transcription factors, several of which govern survival mechanisms. Therefore, the Clp protease machinery substantially contributes to the generalist lifestyle of $B$. subtilis.

\section{S. aureus: Role of Clp in Enabling Survival in the Host and Host Infection}

S. aureus is an important opportunistic human pathogen and can cause severe illnesses like pneumonia, endocarditis, sepsis, or toxic shock syndrome [Balasubramanian et al., 2017; Oliveira et al., 2018]. The excellent adaptive potential of $S$. aureus during colonisation of the human host is based on an arsenal of cell surface-associated and secreted virulence factors. Functions of the Clp system in $S$. aureus range from the general stress response to the regulation of virulence factors (Fig. 2b). Similar to B. subtilis, clpP and $c l p C$ in $S$. aureus are controlled by the heat-inducible CtsR regulon, and both are required for growth at high temperature [Frees et al., 2004]. ClpX expression is not induced upon heat stress and mutants lacking $\operatorname{clp} X$ can grow at high temperature and in the presence of puromycin-induced protein accumulation [Frees et al., 2003; Frees et al., 2004]. The absence of ClpX even results in an improved growth at high temperatures, possibly because increased binding of ClpC to ClpP leads to improved protein quality control [Frees et al., 2003]. The role of ClpXP for protein quality control at elevated temperatures was analysed comparing the growth of $\Delta c l p P, \Delta c l p X$, and $c l p X_{I 256 E}$ mutants. The $\Delta c l p X_{I 256 E} \mathrm{mu}-$ tant cannot form a complex with $\mathrm{ClpP}$ due to an amino acid substitution in the IGF loop of ClpX [Stahlhut et al., 2017]. At $45^{\circ} \mathrm{C}$, the $\Delta c l p X$ and $c l p X_{I 256 E}$ mutants grew as well as the wild-type, while the $\Delta c l p P$ mutant did not grow [Stahlhut et al., 2017]. These results preclude a major role in the heat-shock response for ClpX. However, ClpX seems to be important for growth at decreased temperatures, since deletion of $c l p X$ substantially slowed growth at $30^{\circ} \mathrm{C}$ [Jensen et al., 2019a]. Deletion of $c l p P$ also affected growth at decreased temperatures, but the phenotype was less severe than for $\Delta c l p X$, implying a ClpPindependent function of ClpX in coping with cold-shock [Stahlhut et al., 2017].

There also exists a connection between ClpX and the cell envelope, affecting cell wall hydrolysis and cell division, and the molecular interactions are beginning to emerge. Recently, ClpX was shown to localise close to the division septum, control the cell wall synthesis operon $m r a Z-m r a W-f t s l-p b p A$, and to serve an important function in septum formation and cell division independently of ClpP [Jensen et al., 2019a, b]. Cells lacking $\operatorname{clpX}$ were characterised by septum synthesis arrest and premature splitting of daughter cells at $30^{\circ} \mathrm{C}$, eventually leading to cell lysis [Jensen et al., 2019a]. ClpX mutants showed increased levels of autolysins involved in daughter cell split- 
ting (Sle1 and Atl) and, accordingly, deletion of the autolysins sle 1 and partly also of atl rescued the low-temperature growth defect [Jensen et al., 2019a]. Lipoteichoic acids, which are essential for viability under standard laboratory conditions, became non-essential in cells lacking the ClpX chaperone. Spontaneous loss of function mutations in the ltaS gene (lipoteichoic acid synthase) emerged in the $\mathrm{ClpX}$ mutants at $30^{\circ} \mathrm{C}$ and rescued the growth defect [Bæk et al., 2016]. In a ltaS clpX double mutant, autolysin levels as well as growth were restored to wild-type levels [Bæk et al., 2016]. Interestingly, $\beta$-lactam antibiotics rescued the $\Delta c l p X$ growth defect at low temperature. Oxacillin, for example, allowed septum progression in cells lacking ClpX, thus preventing premature splitting. Also, inhibitors of the first step of wall teichoic acid biosynthesis, i.e., tunicamycin and tarocin A, stimulated the growth of $c l p X$ mutants [Jensen et al., 2019a].

Growth of $S$. aureus at low temperatures is not only supported by ClpX acting as an independent chaperone, but also by the ClpXP protease. The autolysin Sle1 was characterised as a substrate of ClpXP [Feng et al., 2013], and zymograms of cells lacking ClpX and ClpP showed increased Sle1 activity [Bæk et al., 2014]. Furthermore, Atl, which is sequentially cleaved to generate a mature, fully processed version of $62 \mathrm{kDa}$, accumulated in its intermediate form of $85 \mathrm{kDa}$ in cells lacking ClpX and ClpP. Thus, ClpXP was speculated to indirectly affect Atl processing by controlling extracellular proteases [Bæk et al., 2014]. In summary, ClpX chaperone activity is critical for successful septum formation at decreased temperature, and the protease complex ClpXP regulates the stability of cell wall hydrolases, thus controlling the cell wall metabolism.

The overall importance of ClpP in S. aureus is highlighted by the reduced growth rate of $\Delta c l p P$ mutants [Frees et al., 2003; Kirsch et al., 2020], potentially due to reduced levels of proteins involved in purine and pyrimidine biosynthesis [Frees et al., 2012]. A global proteome analysis by Kirsch et al. [2020], aimed to provide a global catalogue of ClpP and ClpX regulated proteins, outlining the effects of a $c l p P$ and a $c l p X$ knockout on the proteome composition. Both mutants were characterised by a strong downregulation of the pur/pyr operon, and metabolomic analyses confirm decreased nucleobase (adenine, cytidine, guanine) and nucleoside (adenosine and guanosine) levels [Kirsch et al., 2020]. Moreover, ClpP affects WalRK-regulated genes [Kirsch et al., 2020], and WalRK is an essential two-component system in $S$. aureus, controlling autolysin activity and cell wall degradation [Dubrac et al., 2007].

Clp Protease in Homoeostasis and Survival
Regarding S. aureus virulence, mutants lacking $c l p P$ or $c l p X$ were incapable of establishing local skin abscesses in mice [Frees et al., 2003]. In a murine model of acute pneumonia, $c l p P$ or $c l p X$ deletion mutants showed a substantially lower bacterial load in the lung, less lung injury, and a reduced inflammatory response [Kim et al., 2020]. As $S$. aureus is the major cause of wound infections in diabetic patients, a global transcription analysis was conducted in diabetic mice to investigate how diabetes influences the infection process [Jacquet et al., 2019]. S. aureus adapted to the hyperglycaemic environment by altering the expression of various genes, among them $c l p P, c l p X$, and $c l p C$, which were all upregulated [Jacquet et al., 2019]. Cells lacking $c l p P$ or $c l p X$ showed decreased $\alpha$-haemolysin production in vitro and reduced dermonecrosis in mice [Jacquet et al., 2019].

Another mechanism by which $S$. aureus adapts to the antagonistic host environment is iron-scavenging from host haemoglobin. ClpXP is important for the expression of the haemoglobin receptor IsdB [Farrand et al., 2013], an essential component of the iron-uptake machinery in $S$. aureus necessary for iron acquisition in the host [Skaar and Schneewind, 2004]. Cells lacking $\operatorname{clpX}$ or $\operatorname{clp} P$ are characterised by reduced $I s d B$ expression under ironpoor conditions [Farrand et al., 2013]. Using immunofluorescence, $\Delta c l p P$ mutants were shown to present less IsdB on the cell surface. Decreased IsdB protein levels could also be detected in immunoblots of $\Delta c l p X$ mutants. Both results correlate with decreased haemoglobin binding of $\Delta c l p P$ and $\Delta c l p X$ mutants, their inability to acquire iron from the host, and reduced bacterial burden in kidneys, livers, and hearts upon systemic infection of mice [Farrand et al., 2013].

Pathogenicity of $S$. aureus depends on the ability to produce a wide range of cell-wall associated and secreted virulence factors, such as protein A, $\alpha$-haemolysin, and extracellular proteases [Lowy, 1998; Frees et al., 2003]. Mutants lacking ClpP or ClpX are characterised by reduced exoproteolytic activity, as well as strongly reduced a-haemolysin secretion [Frees et al., 2003]. The quorum sensing-dependent agr regulatory network is, among others, responsible for virulence factor expression, and induction of the agr operon results in the production of the main effector molecule RNAIII. Cells lacking $c l p P$ or $c l p X$ contained decreased RNAIII levels, implying both as positive regulators of RNAIII [Frees et al., 2003; Michel et al., 2006]. Under physiological conditions, RNAIII accumulates in the post-exponential growth phase and was proposed to interact with Rot (repressor of toxins), resulting in the degradation of Rot by ClpXP and thereby promot- 
ing the expression of the mentioned secreted virulence factors [Frees et al., 2005]. Besides acting as an ATPase partner for regulatory proteolysis of Rot by ClpXP, ClpX on its own was shown to promote the expression of $s p a$, encoding the surface-bound, Fc-receptor scavenger, protein A [Frees et al., 2003, 2005]. ClpX stimulates rot expression, and spa expression is induced upon a certain Rot threshold in the cell. In addition, ClpX stimulates translation of the spa mRNA [Jelsbak et al., 2010]. Besides the widely expressed $\alpha$-toxin and extracellular proteases, a smaller percentage of $S$. aureus strains produces harmful staphylococcal superantigens (SAgs), i.e., the toxic shock syndrome toxin TSST-1, or staphylococcal enterotoxins (SEs) like SEC and SED, causing severe illness [Spaulding et al., 2013]. The gene encoding TSST-1 is tst, which is located on pathogenicity islands (SaPI) [Lindsay et al., 1998]. SEC and SED are mostly associated with food intoxications. SEC is encoded on a SaPI and a plasmid, while SED is solely encoded on a plasmid [Etter et al., 2020]. A study analysing toxin transcript and protein levels in the $\Delta c l p P, \Delta c l p X$, and $c l p X_{I 256 E}$ mutant set concluded that the functional ClpXP protease is required for the induction of $s e c$, sed, and tst in S. aureus clinical isolates, although with strain-specific differences [Schelin et al., 2020]. Moreover, it was shown that ClpXP controls tst expression via a variety of transcriptional regulators, i.e., SarU, SarX, SarT, and MgrA [Schelin et al., 2020]. ClpX was also shown to regulate the expression of further pathogenicity island-encoded genes via ClpP-dependent and ClpP-independent mechanisms [Stahlhut et al., 2017; Jensen et al., 2019b]. For instance, proteins involved in excision and replication of SaPI5 are controlled independently of ClpP [Jensen et al., 2019b]. Furthermore, ClpX controls the expression of the ess locus. The ess locus encodes genes for the type VII secretion machinery as well as secretory proteins, i.e., toxins. The type VII secretion system impacts virulence and is linked to persistent infections caused by $S$. aureus [Burts et al., 2008; Wang et al., 2016; Jensen et al., 2019b].

Treatment of infections caused by $S$. aureus strains using $\beta$-lactams are complicated by the continuous spread of methicillin-resistant $S$. aureus (MRSA) strains [Vestergaard et al., 2019], and the ClpXP protease influences the level of $\beta$-lactam resistance in $S$. aureus. The MRSA strain JE2, like many other community-acquired MRSA strains, displays heterogeneous $\beta$-lactam resistance, with only a small percentage of highly resistant and a larger fraction of low-level resistant cells. Deletion of $c l p P$ or $c l p X$ resulted in a homogenous high-level $\beta$-lactam resistance phenotype in JE2, although the PBP2a level remained constant [Bæk et al., 2014]. A key ClpP substrate in that context is the autolysin Sle1, and cells lacking ClpXP protease activity are characterised by high Sle1 levels and $\beta$-lactam hyper-resistance [Bæk et al., 2014]. In turn, deletion of sle1 in JE2 wild-type cells or in a clp $X_{\text {I265E }}$ mutant incapable of interacting with ClpP led to hyper-susceptibility towards $\beta$-lactams and MIC levels comparable to methicillin-sensitive $S$. aureus strains [Thalsø-Madsen et al., 2019]. In summary, ClpXP controls $\beta$-lactam resistance via degradation of Sle1, which itself strongly contributes to resistance.

Comparing the two protease subtypes ClpXP and Clp$\mathrm{CP}$, it becomes clear that they perform distinct roles in $S$. aureus. ClpXP is the major protease involved in the regulation of the pathogenic lifestyle of S. aureus, while ClpCP plays a major role in protein quality control upon heat stress and under non-stress conditions. In particular, ClpXP is dispensable for degradation of damaged proteins at high temperatures, as cells expressing $\mathrm{ClpX}_{\mathrm{I} 256 \mathrm{E}}$ did not accumulate aggregated proteins upon heat stress [Stahlhut et al., 2017]. In contrast, cells lacking ClpP as well as cells lacking ClpC severely suffer from protein aggregation [Frees et al., 2012; Stahlhut et al., 2017]. Even at $37^{\circ} \mathrm{C}$, protein aggregates appear in $\Delta c l p P$ cells, and the CtsR regulon and the $a h p C$ - $a h p F$ operon, which reacts to oxidative stress, are highly induced in $\triangle c l p P$ mutants. In contrast, there were fewer protein aggregates in cells expressing $\mathrm{ClpX}_{\mathrm{I} 256 \mathrm{E}}$, abolishing ClpXP but retaining ClpCP function [Stahlhut et al., 2017]. This observation points towards a major function of $\mathrm{ClpCP}$ in protein quality control, also under non-stress conditions [Stahlhut et al., 2017]. Although all currently available data distinguish ClpXP as the major virulence-regulating $\mathrm{Clp}$ protease complex in $S$. aureus, there might also be some involvement of ClpCP. This notion is implicated by the observation that $c l p P$ deletion (effecting both $\mathrm{ClpCP}$ and $\mathrm{ClpXP}$ ) resulted in a stronger decrease of agr transcription than the lack of ClpXP protease activity in the $\mathrm{ClpX}_{\text {I256E }}$ mutant [Stahlhut et al., 2017]. Distinct protease roles also correlate with distinct substrate specificities conferred by ClpX or ClpC. Feng et al. [2013] expressed a ClpP ${ }^{\text {TRAP }}$ mutant, which allows trapping of proteins inside the proteolytic chamber without degrading them due to a defective active site. This approach enabled the confirmation of already known substrates as well as the identification of various new $\mathrm{ClpP}$ substrates with regulatory or physiological functions. By expressing the ClpP ${ }^{\mathrm{TRAP}}$ protein in cells lacking either $\mathrm{ClpX}$ or $\mathrm{ClpC}$, different substrate specificities for ClpXP versus ClpCP were determined [Feng et al., 2013]. A variety of substrates were 
identified. Prominent examples of ClpCP substrates were RecA, an important member of the SOS-regulon, the cell division protein FtsZ and GlmS, which catalyses the first step of UDP-N-acetly-D-glucosamine synthesis. Regarding ClpXP substrates, especially Sle1 and Spx were trapped in high amounts [Feng et al., 2013]. Another ClpXP substrate is the SOS-response repressor LexA, which undergoes autocleavage upon DNA stress. The resulting N-terminal domain is mainly degraded by ClpXP, but $\mathrm{ClpCP}$ appears to contribute to the degradation. In contrast, the C-terminal domain was not degraded by Clp proteases [Cohn et al., 2011].

The Clp protease also affects biofilm formation by $S$. aureus, with $c l p P$ deletion increasing and $c l p X$ and $c l p C$ deletions decreasing the amount of biofilm formed. However, the molecular explanation is still elusive [Frees et al., 2004]. Last but not least, ClpCP degrades antitoxins in $S$. aureus. Toxin-antitoxin systems are small genetic elements encoding a toxin and its corresponding antitoxin, and upon environmental stress toxin production is enhanced. In S. aureus, MazF, for example, encodes an RNase toxin responsible for decreased growth rates as well as decreased hla and spa amounts [Schuster and Bertram, 2016]. MazE represents the corresponding antitoxin. Besides MazEF, S. aureus also produces the axe1-txe1 and axe2-txe 2 toxin-antitoxin system. ClpCP was shown to degrade all three antitoxins [Donegan et al., 2010].

Taken together, the Clp system exerts a strong impact on the physiology of $S$. aureus, promoting colonisation and infection in the host environment in a manifold manner. ClpXP and ClpCP have a distinct focus, with multiple functions in virulence regulation for the former, and a prominent role in protein quality control for the latter. For clarification, two additional Clp-ATPases, ClpB and $\mathrm{ClpL}$, are present in $S$. aureus. However, they lack the consensus tripeptide motif essential for the interaction with ClpP [Kim et al., 2001; Frees et al., 2004]. Therefore, they act as independent chaperones but are not further discussed in this review, as they do not provide substrates to the conserved proteolytic core. S. aureus is featured in this review as a particularly well-studied example of the impact of the Clp protease system on bacterial virulence. Notably, $c l p P$ deletion was also shown to attenuate the virulence of other clinically relevant species like Staphylococcus epidermidis, Streptococcus pneumoniae, or L. monocytogenes [Gaillot et al., 2000, 2001; Kwon et al., 2004; Wang et al., 2007].

Clp Protease in Homoeostasis and Survival

\section{M. tuberculosis, L. monocytogenes, C. trachomatis: Importance of Clp for a Facultative or Obligate Intracellular Lifestyle}

M. tuberculosis is a rod-shaped, acid-fast, and facultative intracellular human pathogen. The lifecycle is characterised by two different metabolic states, one of active replication and a non-proliferative, persistent state [Glickman and Jacobs, 2001]. Switching between these lifestyles and ensuring extracellular as well as intracellular survival, demands high proteome plasticity. M. tuberculosis contains two ClpP homologs encoded in a bicistronic operon, with both $c l p P 1$ and $c l p P 2$ positioned downstream of $c l g R$, the conserved activator of $c l p P$ expression in Actinobacteria [Personne et al., 2013]. Biochemical and structural studies demonstrated that the functional ClpP complex of $M$. tuberculosis is a hetero-tetradecamer composed of a ClpP1 and a ClpP2 homo-heptameric ring, designated ClpP1P2 and meaning that one ring is made of seven ClpP1 subunits, while the heptamer stacked to it exclusively contains ClpP2 [Ingvarsson et al., 2007; Akopian et al., 2012; Ollinger et al., 2012]. Although originating from co-transcribed genes, ClpP1 and ClpP2 are surprisingly dissimilar in their amino acid sequence, and both mycobacterial ClpP homologs share relatively low sequence identities with $\mathrm{ClpP}$ proteins from other bacteria (Fig. 3).

It is important to note that, unlike most bacteria, both mycobacterial ClpP homologs are essential for M. tuberculosis, even under standard growth conditions in culture broth in vitro [Ollinger et al., 2012]. Reduction of functional mycobacterial ClpP1P2 via clpP knock-down or active site mutagenesis resulted in severe attenuation in a mouse lung infection and deficiency to replicate within macrophages [Carroll et al., 2011; Raju et al., 2012]. Although it remains to be elucidated which Clp-ATPase is involved and how the regulatory network is arranged, mycobacterial ClpP1P2 was reported to degrade its regulator ClgR (Fig. 2c). Other strong candidates for Clp-mediated degradation include essential transcription factors such as WhiB1 and CarD, accumulation of which proved to be detrimental or even fatal for M. tuberculosis, offering a rationale for the essentiality of the Clp protease in this species [Raju et al., 2014; Yamada and Dick, 2017].

In vitro studies on the degradation of substrates by purified M. tuberculosis ClpP1P2 are only just emerging. One group of substrates demonstrated to be digested by $\mathrm{ClpP} 1 \mathrm{P} 2$ are ssrA-tagged proteins. Such proteins are released from the ribosome in the process of trans-translation. For ssrA-protein degradation, mycobacterial ClpX 
Fig. 3. Phylogenetic clustering of the ClpP proteins discussed in this review. a ClpP protein homology analysis based on CLANS (cluster analysis of sequences). A total of 597 unique sequences were extracted from the Swiss-Prot database, and ImClpP1 (Q8Y7Y1) was extracted from the TrEMBL database. The $p$ value threshold was set to 1e-81. Atypical ClpP species such as ctClpP1 (P38002), lmClpP1 (Q8Y7Y1), mtClpP1 (P9WPC5), and mtClpP2 (P9WPC3) are highlighted. $\mathbf{b}$ Zoomed view of the large cluster comprising over $70 \%$ (437/598) of total input sequences. Most ClpP species are highly conserved, including saClpP (P63786), bsClpP (P80244), and lmClpP2 (Q9RQI6). Human mitochondrial hsClpP (Q16740) and ctClpP2 (O84712) are located at the margin of the highly conserved ClpP cluster, outlining distinct sequence variation. bs, B. subtilis; ct, C. trachomatis; hs, Homo sapiens, $\mathrm{lm}$, L. monocytogenes; $\mathrm{mt}$, M. tuberculosis; sa, S. aureus.

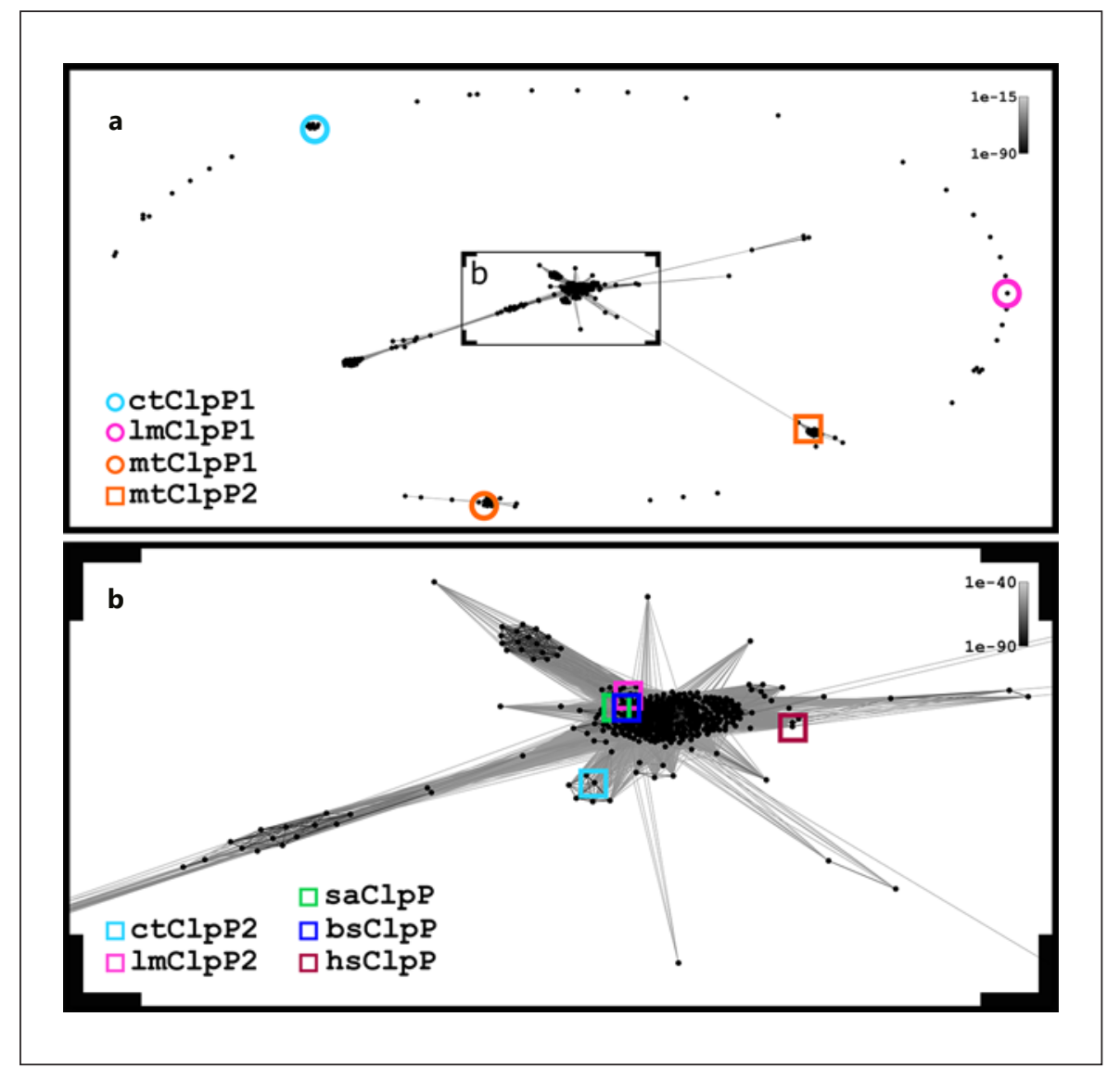

and $\mathrm{ClpC} 1$ both interact exclusively with $\mathrm{ClpP} 2$ and not with ClpP1 [Leodolter et al., 2015; Nagpal et al., 2019]. Recently, ClpC1 has been reported to be heavily involved in the regulation of a myriad of toxin-antitoxin systems [Ziemski et al., 2021]. When the isolated M. tuberculosis ClpP1P2 core was studied, it emerged that catalytic activity was strongly stimulated by the addition of certain activator peptides, which did not act as substrates but stabilised the catalytically competent conformation of the active sites [Akopian et al., 2012; Liu and Chien, 2014; Schmitz et al., 2014; Famulla et al., 2016]. A cryo-EM study recently revealed that this characteristic feature of M. tuberculosis $\mathrm{ClpP}$ is caused by the absence of a typical $\beta$-sheet within the handle region of $\mathrm{ClpP}$ and the resulting flexibility in this region that shifts the tetradecamer into an inactive compact conformation [Vahidi et al., 2020]. The peptide agonists increase order in this region of ClpP and restore the active extended conformation of the barrel. It is assumed that within the cell peptide products serve this agonistic function, generated by a Clp-ATPase feeding an unfolded polypeptide through the entrance pores of ClpP [Schmitz and Sauer, 2014; Schmitz et al., 2014]. As the interaction of separate ClpP proteins, i.e., ClpP1 plus ClpP2, plus product activation seems to be required for an active proteolytic core, the mycobacterial Clp protease appears particularly tightly regulated [Raju et al., 2012; Schmitz et al., 2014; Alhuwaider and Dougan, 2017].

L. monocytogenes is a facultative intracellular pathogen with two distinct $c l p P$ genes, which, unlike M. tuberculosis, are not co-transcribed. Among the ClpPs of Listeria, ClpP2 is very similar to the ClpP proteins found in single-ClpP organisms and possesses the canonical SerHis-Asp catalytic triad, whereas ClpP1 shares only $41 \%$ identity with ClpP2 (Fig. 3), and in the catalytic triad of ClpP1 the conserved Asp is exchanged for Asn [Zeiler et al., 2011, 2013]. Active-site directed labelling with various $\beta$-lactone suicide inhibitors, size exclusion chromatography, and peptidase activity assays found ClpP2 of $L$. monocytogenes to form an active tetradecameric peptidase, while ClpP1 alone adopted only an inactive heptamer that resisted $\beta$-lactone labelling [Böttcher and 
Sieber, 2008; Zeiler et al., 2011]. However, upon co-expression or mixing of both homologs, ClpP2 activated ClpP1 by hetero-oligomerisation [Zeiler et al., 2011; Dahmen et al., 2015]. In the resulting ClpP1P2 hetero-complex, two homo-heptamers of exclusively $\mathrm{ClpP} 1$ and ClpP2 are stacked onto each other, and all 14 catalytic residues within both ClpP subunits are correctly aligned for catalysis, greatly enhancing its proteolytic capabilities [Dahmen et al., 2015]. In corroboration of this finding, in the presence of cognate $\mathrm{ClpX}$, the proteolytic activity of the ClpP1P2 hetero-complex was 10-fold higher than that of the ClpP2 homo-tetradecamer [Dahmen et al., 2015].

The importance of Clp proteins in Listeria pathogenesis is conspicuous. While the physiological function of $\mathrm{ClpP} 1$ remains largely elusive so far, the deletion of $c l p P 2$ is fatal for L. monocytogenes during intracellular growth in macrophages and rendered the mutant avirulent in mice [Gaillot et al., 2000; Gaillot et al., 2001]. This lack of intracellular survival has been linked to the depletion of the Listeria-specific haemolysin listeriolysin O (Fig. 2d), a major virulence factor responsible for phagosome escape during Listeria infection [Gaillot et al., 2000]. As for its corresponding Clp-ATPases, ClpX has been shown to mediate the degradation of ssrA-tagged proteins by ClpXP1P2 in vitro, and knock-out mutagenesis of $c l p C$ and $c l p E$ revealed that the lack of either or both chaperones caused pleiotropic physiological impairments, including deficiencies in cell division, general stress tolerance, and virulence [Nair et al., 1999, 2000; Balogh et al., 2017].

Chlamydia trachomatis is a Gram-negative obligate intracellular bacterium with an obligatory biphasic, parasitic lifestyle that preferably infects human epithelial cells. C. trachomatis encodes two ClpP homologs, which are not co-transcribed and only $\mathrm{ClpP} 2$ shares a relatively high sequence identity with most other ClpP homologs (Fig. 3). In terms of the ClpP oligomer organisation, the chlamydial ClpP proteins are different insofar as ClpP1 as well as ClpP2 formed only heptamers in vitro. Consequently, both subunits are required for the assembly of the functional hetero-tetradecamer. The fact that the two $\operatorname{clp} P$ genes of chlamydia are encoded in separate chromosomal locations, although their protein products strictly depend on each other for catalytic function, suggests a safeguard mechanism on the genomic level, only granting protease action when correct transcription is ensured [Pan et al., 2019]. In biochemical studies, the active sites of ClpP 1 and $\mathrm{ClpP} 2$ contributed about equally to the total catalytic rate of the proteolytic core, while ClpX interacted only with ClpP2 [Pan et al., 2019].

Clp Protease in Homoeostasis and Survival
C. trachomatis is an obligate parasite and possesses a very reduced genome size of only about $1 \mathrm{Mb}$. Genome comparison reveals that many intracellular proteolytic systems known from free-living bacteria, such as DegS(HtrH), HslUV(ClpQY), Thr/Cys proteases and the prokaryotic proteasome, are not available, which emphasises the contribution of the remaining ones. In the unique chlamydial lifecycle, alternating between infectious elementary bodies (EB) and replicative reticulate bodies (RB), proteome plasticity is essential and unimpeded Clp function is expected to play a critical role in ensuring protein turnover during cell development and pathogenesis [Rodgers et al., 2010; Pan et al., 2019; Wood et al., 2020]. Degradation of ssrA-tagged proteins by chlamydial ClpXP1P2 has been demonstrated in recent studies, both in vitro and in intracellular chlamydia (Fig. 2e) [Pan et al., 2019; Wood et al., 2020]. Genetic manipulation of the obligate intracellular pathogen is challenging and has been managed only as of late. In a recent study by Wood et al. [2020], the authors attempted to disrupt the functionality of the protease system by overexpressing mutated, inactive ClpX and ClpP2 variants within chlamydial cells. Titrating the defective proteins against the remaining wildtype forms is expected to result in non-functional mosaic complexes and yield a knock-down phenotype. Overexpression of inactive $\mathrm{ClpX}$ or $\mathrm{ClpP} 2$ led to a sharp drop in recoverable inclusion-forming units (i.e., released infectious EB particles), although likely due to different physiological reasons. The authors propose a model in which $\mathrm{ClpP} 2$ is required for the transition of the $\mathrm{EB}$ to $\mathrm{RB}$ state in the developmental cycle and ClpX for reproduction and infectivity of the EBs [Wood et al., 2020]. Natural substrates and regulatory proteolysis of the C. trachomatis Clp protease are the subject of current research, including the role of ClpP1. The observation that M. tuberculosis, L. monocytogenes, and C. trachomatis contain hetero-tetradecameric $\mathrm{ClpP}$ cores, engaging only $\mathrm{ClpP} 2$ in asymmetric interactions with the Clp-ATPases investigated so far, implies an expanded regulatory capacity, which might be crucial for survival or intracellular persistence [Leodolter et al., 2015; Gatsogiannis et al., 2019].

\section{Mitochondrial Clp: Bacteria-Derived Protease Mediating Protein Homoeostasis in Eukaryotic Cells}

The importance of the Clp machinery manifests in the high diversity of bacteria relying on this system, and the Clp protease was probably one of the benefits, the a-protobacterium that later evolved to mitochondria, 
brought into the proto-eukaryotic cell [Valera-Alberni and Canto, 2018]. In recent eukaryotic cells, the mitochondrial Clp protease makes an important contribution to protein quality control and homoeostasis as well as the regulation of oxidative phosphorylation (OXPHOS), mitochondrial ribosome biogenesis, various stress response pathways and signalling cascades (Fig. 2f) [Voos, 2013; Valera-Alberni and Canto, 2018]. Although only the mitochondrial Clp protease will be discussed further in this review, it should be mentioned that also two further organelles contain a Clp protease, i.e., chloroplasts and the apicoplast of the parasitic phylum Apicomplexa [Yu and Houry, 2007; Bhandari et al., 2018].

The metabolism of the eukaryotic cell depends on an immaculate mitochondrial function at all times. Besides their prominent role in bioenergetics by performing the tricarboxylic acid cycle and OXPHOS, mitochondria are essential for amino acid and fatty acid metabolism, steroid hormone production, and cofactor synthesis [Voos, 2013]. They serve as a signalling hub for various stress responses, innate immunity and apoptotic pathways, perform central functions in cell cycle regulation and cell differentiation, and mitochondrial dysfunction is a central factor in cellular senescence [Antico Arciuch et al., 2012; Voos, 2013; Sun et al., 2016]. To ensure functional integrity, mitochondrial protein homoeostasis is mandatory and maintained by a set of chaperones and proteases interconnected in a functional network. The Clp protease is one of three major proteases responsible for protein turnover in the mitochondrial matrix [Calvo and Mootha, 2010; Voos, 2013].

The genes of human mitochondrial $c l p P$ and $c l p X$ are encoded in the nucleus on chromosome 19 and 15, respectively, and translated as precursor proteins with an N-terminal mitochondrial targeting sequence to be removed upon uptake into the organelle [Kang et al., 2002]. Human mitochondrial ClpP is highly homologous to its bacterial equivalents, in sequence as well as in structure [Kang et al., $2002,2004]$. The proteolytic core is composed of a single ClpP homolog, and ClpX is the only Clp-ATPase present [Kang et al., 2002, 2004]. Structural and functional conservation allowed an interaction of mitochondrial ClpP with E. coli ClpX, resulting in a functional complex, capable of recognising $E$. coli-specific substrates and their degradation tags, like the ssrA-tag [Kang et al., 2004]. In contrast, human mitochondrial ClpXP could not process ssrA-tagged substrates in vitro, while the model substrate casein was efficiently degraded [Kang et al., 2004].

Efforts to elucidate natural substrates and regulation networks of mitochondrial ClpXP have only just begun
[Fischer et al., 2015; Wong and Houry, 2019]. Several potential substrates and interaction partners were recently proposed, based on proteins accumulating in $c l p P$ and/or clpX deletion mutants or ClpP/ClpX pull-down experiments and further candidates are discussed based on human homologs of bacterial proteins [Cole et al., 2015; Fischer et al., 2015; Seo et al., 2016]. To our knowledge, the only substrate verified in vitro is NOA1, an essential mitochondrial GTPase involved in the regulation of mitochondrial respiration and mitochondrial ribosome biogenesis [Heidler et al., 2011; Kolanczyk et al., 2011; AlFuroukh et al., 2014]. Further ClpXP substrates deduced from proteomics and physiological investigations in whole-cell studies include SDHB and ERAL1 [Seo et al., 2016; Szczepanowska et al., 2016]. SDHB is the iron sulphur subunit B of the succinate dehydrogenase complex required for a functional respiratory complex II, and its misfolding and accumulation in the absence of ClpXP disrupts the electron transport chain in mitochondria [Seo et al., 2016]. ERAL1 is a putative $12 \mathrm{~S}$ rRNA chaperon, the timely removal of which from the small ribosomal subunit is essential for the maturation of mitochondrial ribosomes [Szczepanowska et al., 2016]. SDHA, subunit $\mathrm{A}$ of the succinate dehydrogenase, and elongation factor EFG1 also accumulated in clp deletion mutants, further substantiating the role of ClpXP in mitochondrial bioenergetics and translation [Szczepanowska et al., 2016; Mirali and Schimmer, 2020]. Additional discussed substrates include proteins involved in amino acid and fatty acid metabolism, the pyruvate dehydrogenase complex, protein import as well as chaperones [Cole et al., 2015; Fischer et al., 2015; Deepa et al., 2016; Seo et al., 2016; Szczepanowska et al., 2016].

Most networks of regulatory proteolysis involving ClpXP in mitochondria are still elusive and an active field of research. The best-understood participation of the Clp protease in a pathway is the mitochondrial unfolded protein response (UPR $\left.{ }^{\mathrm{mt}}\right)$ in the model organism Caenorhabditis elegans. $\mathrm{UPR}^{\mathrm{mt}}$ represents the primary signalling pathway for restoration of protein balance in mitochondria and occurs when defective proteins accumulate in the matrix, import of proteins into mitochondria is hampered, or if the established stoichiometry between proteins encoded in the nucleus and proteins encoded in the mitochondria is disturbed [Pellegrino et al., 2013]. Several factors signal protein stress to the nucleus, among them proteins, the uptake of which into the matrix fails, and ClpXP, which generates peptide fragments that are exported into the cytoplasm. In response, nucleus-encoded chaperones and proteases are upregulated and import- 
ed into the mitochondria to re-establish protein homoeostasis. In mammalian cells, ClpXP also plays an important role in reducing protein stress in the mitochondrial matrix by degrading defective proteins and its level is elevated during the UPR ${ }^{\mathrm{mt}}$ [Al-Furoukh et al., 2015; Deepa et al., 2016; Seiferling et al., 2016]. Moreover, ClpXP was shown to be involved in the turnover of the mitophagy kinase PINK1 [Greene et al., 2012; Pellegrino et al., 2013]. PINK1 accumulates on the outer membrane of defective mitochondria, recruiting the ubiquitin ligase Parkin and causing the activation of the downstream autophagy machinery to direct the functionally dead organelle to lysosomes. Furthermore, there are hints suggesting a contribution of the Clp protease in mitochondrial fusion and fission dynamics, as mitochondria of clpP knock-down cells were smaller and more scattered throughout the cell and the dynamin-related protein Drp1 accumulated, which is known to induce fission [Deepa et al., 2016].

The ClpXP protease is almost omnipresent in eukaryotic cells, but different cell types seem to depend on it to a different extent. A strong expression of human mitochondrial ClpP was observed in skeletal muscle followed by heart, liver, and pancreas, while significantly lower expression levels were determined in the brain, placenta, lung, and kidney [Bross et al., 1995; Wong and Houry, 2019]. In a myoblast cell line, knock-down of mitochondrial ClpP resulted in impaired proliferation and trigged a failure to differentiate into myotubes, while the mitochondria showed morphological defects, a reduced respiration rate, decreased membrane potential, and increased ROS production [Deepa et al., 2016]. Dysfunction of human mitochondrial ClpP can also lead to human disease. A direct link is established for the Perrault syndrome, a rare human recessive genetic disorder, causing severe sensorineural hearing loss, neurological defects, and developmental delay as well as ovarian dysfunction in females [Gispert et al., 2013; Bhandari et al., 2018]. Different gene defects in six distinct genes were identified to cause the Perrault syndrome (types 1-6 respectively), among them $c l p P$ mutations (Perrault syndrome type 3 ). Seven different point mutations in the $c l p P$ gene were identified in patients, impairing the docking site of the interacting Clp ATPase or the catalytic triad and one mutation even both in parallel [Brodie et al, 2018]. The defect results in an unstable ClpP protein with an impaired structure and shorter half-life, leading to overall mitochondrial dysfunction [Ahmed et al., 2015; Dursun et al., 2016; Bhandari et al., 2018]. In the neurogenerative disease Friedrich ataxia (FRDA), ClpP upregulation was attributed to disease progression, although indirectly, as the genetic defect resides in the gene encoding the mitochondrial protein frataxin, an iron chaperone contributing to the biosynthesis of $\mathrm{Fe}-\mathrm{S}$ clusters in mitochondria [Guillon et al., 2009]. Loss of frataxin function results in low amounts of $\mathrm{Fe}-\mathrm{S}$ proteins in mitochondria and other compartments, and consequently reduced OXPHOS performance and elevated ROS levels [Guillon et al., 2009; Fischer et al., 2015]. In a study using a mouse model for FRDA, ClpP and the protease Lon were both upregulated during the middle stage of the disease, in parallel with a further decrease of $\mathrm{Fe}-\mathrm{S}$ proteins, and $\mathrm{Fe}-\mathrm{S}$ protein proteolysis was hypothesised [Guillon et al., 2009].

\section{Mitochondrial Clp in Cancer Cells: Capitalising on a Bacterial System for Survival under Stress}

The characteristic unrestrained growth and proliferation of cancer cells make cell homoeostasis a particular challenge. Extensive reprogramming of cellular metabolism, as a direct or indirect consequence of tumorigenic mutations, is a hallmark of cancer cells. It involves major modifications in energy metabolism (e.g., substantially increased glycolysis and lactic-acid fermentation), alternative precursor flows in anabolism (e.g., more biosynthetic precursors derived from glycolysis), and versatile, opportunistic routes of nutrient acquisition, to name just a few [Pavlova and Thompson, 2016]. Contrary to a previous notion that cancer cells would generate ATP predominately by glycolysis, recent studies indicate that, despite their heavy fermentation, most tumours still rely on mitochondrial OXPHOS for ATP generation and that mitochondria serve multiple central functions in tumour cell progression, including protein homoeostasis and quality control [DeBerardinis and Chandel, 2016; Zong et al., 2016]. The requirement for constant nutrient and ATP replenishment due to unchecked growth, elevated ROS levels due to increased metabolic rates, acidosis due to fermentation, and hypoxia due to increased oxygen demand or during metastatic dissemination, all represent factors that impose stress on tumour cells. Cancer cells have developed multifarious strategies to alleviate the stress, and with regard to preserving mitochondrial function, multiple chaperones and proteases are upregulated, including ClpXP.

In about half of all patients with primary acute myeloid leukaemia (AML), ClpP was overexpressed, suggesting a role in pathology [Cole et al., 2015]. A protein-protein interaction study in AML cells yielded SDHA as a primary interaction partner of $\mathrm{ClpP}$, in agreement with reduced 
respiratory complex II activity, oxygen consumption, and viability of the cells upon $\operatorname{clpP}$ knock-down [Cole et al., 2015]. Notably, these inhibitory effects were only observed in AML subtypes distinguished by ClpP overexpression, whereas AML cells with a normal ClpP amount were not affected, raising hopes for $\mathrm{ClpP}$ as a target in subtypes of cancer with an elevated ClpP amount. Apart from AML, ClpP levels were also found increased in other hematologic malignancies as well as solid prostate tumours and sarcomas [Cole et al., 2015]. In a study with mitochondria from prostate carcinoma cells, immunoprecipitation experiments suggested the formation of a complex between ClpXP, the Hsp90-like chaperone TRAP-1, and the oncoprotein survivin [Seo et al., 2016]. Survivin and ClpXP also interacted with the respiratory II complex subunit SDHB. The authors propose that a ClpXP-survivin-TRAP-1 complex acts as a protein quality control system, combining the refolding capability of the chaperone(s) and proteolytic activity of the protease, and that the complex supports SDHB function, thereby contributing to the regulation of respiration. Knock-down of ClpXP resulted in an accumulation of misfolded SDHB, impaired OXPHOS, oxidative stress, reduced tumour cell proliferation, and inhibition of metastasis [Seo et al., 2016]. These examples show that certain tumours can exploit ClpXP-mediated protein homoeostasis to maintain mitochondrial function and tumour cell survival under stress.

\section{Conclusion}

Bacterial survival strongly depends on proteome flexibility to enable adaptation and on proteome homoeostasis to safeguard essential functions. Protein quality control helps to cope with protein damage under stress, and targeted proteolysis of central regulatory factors (often transcription factors) enables a response to changing environmental conditions. In that general regard, there are strong parallels between all bacterial species featured in this review, and also between bacteria and mitochondria, and in all of them the Clp protease machinery makes essential contributions to both the maintenance of protein homoeostasis and targeted regulatory proteolysis. Even in bacteria, like Firmicutes, where Clp protease function is dispensable under non-stressing growth conditions in vitro (i.e., in the presence of sufficient nutrients, at moderate temperatures, and in the absence of inhibiting agents), the Clp protease becomes essential, when stress increases (e.g., when bacteria enter the stationary phase or the host environment). By pairing the central ClpP core with different cognate Clp-ATPase partners, not only flexibility in the selection of proteolytic substrates is generated, but it is also guaranteed that the options of protein refolding and protein turnover are intricately linked.

Important specific contributions of the Clp protease system to bacterial survival range from the degradation of defective, misfolded, or aggregated proteins and the general rescue of stalled ribosomes to precise regulatory control of the heat-shock response, natural competence, sporulation, motility, virulence, and lifecycle management. In the well-studied system of $B$. subtilis, it emerged that in addition to the proteolytic core $\mathrm{ClpP}$ and the different cognate Clp-ATPases, a variety of adaptor proteins fine tune the proteolytic response. It also became clear that by interacting with one versus the other of its ClpATPase partners, ClpP can either promote or inhibit a given development process. Rather as a rule than an exception, the Clp protease also recognises its proteolytic activators or repressors as substrates generating an elaborate and entwined network of regulatory control. It is to be expected that in species, where investigations on the Clp machinery have commenced more recently and where only the rudiments of substrates and interaction partners are known so far, similar regulatory layers await discovery. The asymmetric ClpP1P2 cores discovered in several bacterial species so far, including the intracellular species discussed here, offer further regulatory potential, as the assembly of two different ClpP homologs, sometimes even expressed from distinct chromosomal locations, are required for catalytic function. Also, eukaryotic cells capitalise from the capacity of the Clp protease to alleviate protein stress and perform regulatory proteolysis to ensure proper mitochondrial function and cellular homoeostasis.

Due to its prominent role in regulating bacterial survival and virulence as well as proliferation and metastasis of cancer cells, the Clp protease holds promise as an antibiotic and anti-tumour drug target. Adding to this is the fact that the protease proved to be druggable by more than one compound class and that its inhibition as well as activation showed therapeutic potential. Acyldepsipeptides of the ADEP class dysregulate $\mathrm{ClpP}$ by inhibiting its natural functions, yet at the same time stimulating unregulated proteolysis of non-native substrates [Kirstein et al., 2009a; Famulla et al., 2016]. ADEP has broad-spectrum anti-Gram-positive antibiotic activity, displaying low minimal inhibitory concentrations and potent in vivo efficacy [Brötz-Oesterhelt et al., 2005; Brötz-Oesterhelt and Vorbach, 2021]. The specific inhibition of the 
Clp protease is likewise promising and pursued as an anti-virulence strategy. $\beta$-Lactones and the dihydrothiazepine-derivative 334 represent $\mathrm{ClpP}$ and ClpX inhibitors, respectively. $\beta$-Lactones were shown to attenuate $S$. aureus in mouse infections, and 334 was reported to attenuate global virulence factor synthesis in vivo [Weinandy et al., 2014; Fetzer et al., 2017]. Cyclomarin, lassomycin, and ecumicin are antibacterial agents that deregulate the Clp-ATPase component of the Clp protease complex [Malik and Brötz-Oesterhelt, 2017]. Regarding anti-tumourigenic potential, ADEP derivatives showed some potential, and for the imipridone ONC 201, a first-inclass anticancer compound in clinical trials, $\mathrm{ClpP}$ has recently been established as the target [Wong et al., 2018; Graves et al., 2019; Ishizawa et al., 2019; Wong and Houry, 2019].

The ubiquitous Clp protease system represents an especially versatile machinery, involved in various cellular homoeostasis processes and coping strategies. It will be fascinating to unravel additional functions in bacteria and eukaryotic cells and further explore its therapeutic potential.

\section{Acknowledgements}

The authors thank their coworkers, collaborators, and all members of the GRK1708 for helpful discussions.

\section{Conflict of Interest Statement}

The authors have no conflicts of interest to declare.

\section{Funding Sources}

We gratefully acknowledge financial support from the German Research Foundation (DFG). Funding was provided by the GRK1708 (to A.I., L.R., and H.B.-O.) and the TRR261, project ID 398967434 (to S.P., Y.T., and H.B-O.).

\section{Author Contributions}

A.I. drafted the passages on B. subtilis and S. aureus, prepared Figure 2, and assembled the final document. Y.T. drafted the passages on mitochondrial ClpP. S.P. drafted the passages on L. monocytogenes, M. tuberculosis, and C. trachomatis, and prepared Figure 3. L.R. drafted the passage on the introduction to the Clp system and prepared Figure 1. H.B-O. devised the concept and structure of this review and finalised the text.

\section{References}

Ahmed S, Jelani M, Alrayes N, Mohamoud HS, Almramhi MM, Anshasi W, et al. Exome analysis identified a novel missense mutation in the CLPP gene in a consanguineous Saudi family expanding the clinical spectrum of Perrault syndrome type-3. J Neurol Sci. 2015 Jun;353(1-2):149-54.

Akopian T, Kandror O, Raju RM, Unnikrishnan M, Rubin EJ, Goldberg AL. The active ClpP protease from $\mathrm{M}$. tuberculosis is a complex composed of a heptameric ClpP1 and a ClpP2 ring. EMBO J. 2012 Mar;31(6):1529-41.

Al-Furoukh N, Kardon JR, Krüger M, Szibor M, Baker TA, Braun T. NOA1, a novel ClpXP substrate, takes an unexpected nuclear detour prior to mitochondrial import. PLoS One. 2014 Jul;9(7):e103141.

Al-Furoukh N, Ianni A, Nolte H, Hölper S, Krüger $\mathrm{M}$, Wanrooij S, et al. ClpX stimulates the mitochondrial unfolded protein response (UPRmt) in mammalian cells. Biochim Biophys Acta. 2015 Oct; 1853(10):2580-91.

Alhuwaider AAH, Dougan DA. AAA+ machines of protein destruction in mycobacteria. Front Mol Biosci. 2017 Jul;4:49.

Antico Arciuch VG, Elguero ME, Poderoso JJ, Carreras MC. Mitochondrial regulation of cell cycle and proliferation. Antioxid Redox Signal. 2012 May;16(10):1150-80.
Bæk KT, Gründling A, Mogensen RG, Thøgersen L, Petersen A, Paulander W, et al. $\beta$-Lactam resistance in methicillin-resistant Staphylococcus aureus USA300 is increased by inactivation of the ClpXP protease. Antimicrob Agents Chemother. 2014 Aug;58(8):4593603.

Bæk KT, Bowman L, Millership C, Dupont Søgaard M, Kaever V, Siljamäki P, et al. The cell wall polymer lipoteichoic acid becomes nonessential in Staphylococcus aureus cells lacking the ClpX chaperone. mBio. 2016 Sep; 7(4):e01228-16.

Balasubramanian D, Harper L, Shopsin B, Torres VJ. Staphylococcus aureus pathogenesis in diverse host environments. Pathog Dis. 2017 Jan;75(1):ftx005.

Balogh D, Dahmen M, Stahl M, Poreba M, Gersch $\mathrm{M}$, Drag $\mathrm{M}$, et al. Insights into ClpXP proteolysis: heterooligomerization and partial deactivation enhance chaperone affinity and substrate turnover in Listeria monocytogenes. Chem Sci. 2017 Feb;8(2):1592-600.

Bhandari V, Wong KS, Zhou JL, Mabanglo MF, Batey RA, Houry WA. The Role of ClpP protease in bacterial pathogenesis and human diseases. ACS Chem Biol. 2018 Jun;13(6): 1413-25.

Böttcher T, Sieber SA. $\beta$-Lactones as privileged structures for the active-site labeling of versatile bacterial enzyme classes. Angew Chem Int Ed. 2008 May;47(24):4600-3.
Brito PH, Chevreux B, Serra CR, Schyns G, Henriques AO, Pereira-Leal JB. Genetic competence drives genome diversity in Bacillus subtilis. Genome Biol Evol. 2018 Jan;10(1):10824.

Brodie EJ, Zhan H, Saiyed T, Truscott KN, Dougan DA. Perrault syndrome type 3 caused by diverse molecular defects in CLPP. Sci Rep. 2018;8(1):12862.

Bross P, Andresen BS, Knudsen I, Kruse TA, Gregersen N. Human ClpP protease: cDNA sequence, tissue-specific expression and chromosomal assignment of the gene. FEBS Lett. 1995 Dec;377(2):249-52.

Brötz-Oesterhelt H, Beyer D, Kroll HP, Endermann R, Ladel C, Schroeder W, et al. Dysregulation of bacterial proteolytic machinery by a new class of antibiotics. Nat Med. 2005 Oct; 11(10):1082-7.

Brötz-Oesterhelt H, Sass P. Bacterial caseinolytic proteases as novel targets for antibacterial treatment. Int J Med Microbiol. 2014 Jan; 304(1):23-30.

Brötz-Oesterhelt H, Vorbach A. Reprogramming of the caseinolytic protease by ADEP antibiotics: molecular mechanism, cellular consequences, therapeutic potential. Front $\mathrm{Mol}$ Biosci. 2021 May;8:690902. 
Burts ML, Dedent AC, Missiakas DM. EsaC substrate for the ESAT-6 secretion pathway and its role in persistent infections of Staphylococcus aureus. Mol Microbiol. 2008 Aug; 69(3): 736-46.

Calvo SE, Mootha VK. The mitochondrial proteome and human disease. Annu Rev Genomics Hum Genet. 2010 Sep;11(1):25-44.

Carroll P, Faray-Kele MC, Parish T. Identifying vulnerable pathways in Mycobacterium tuberculosis by using a knockdown approach. Appl Environ Microbiol. 2011 Jul;77(14):5040-3.

Cohn MT, Kjelgaard P, Frees D, Penadés JR, Ingmer H. Clp-dependent proteolysis of the LexA N-terminal domain in Staphylococcus aureus. Microbiology. 2011 Mar; 157(Pt 3): $677-84$.

Cole A, Wang Z, Coyaud E, Voisin V, Gronda M Jitkova $Y$, et al. Inhibition of the mitochondrial protease $\mathrm{ClpP}$ as a therapeutic strategy for human acute myeloid leukemia. Cancer Cell. 2015 Jun;27(6):864-76.

D’Souza C, Nakano MM, Zuber P. Identification of comS, a gene of the srfA operon that regulates the establishment of genetic competence in Bacillus subtilis. Proc Natl Acad Sci. 1994 91(20):9397-401.

Dahmen M, Vielberg MT, Groll M, Sieber SA. Structure and mechanism of the caseinolytic protease $\mathrm{ClpP} 1 / 2$ heterocomplex from Listeria monocytogenes. Angew Chem Int Ed Engl. 2015 Mar;54(12):3598-602.

DeBerardinis RJ, Chandel NS. Fundamentals of cancer metabolism. Sci Adv. 2016 May;2(5): e1600200.

Deepa SS, Bhaskaran S, Ranjit R, Qaisar R, Nair BC, Liu Y, et al. Down-regulation of the mitochondrial matrix peptidase $\mathrm{ClpP}$ in muscle cells causes mitochondrial dysfunction and decreases cell proliferation. Free Radic Biol Med. 2016 Feb;91:281-92.

Derre I, Rapoport G, Msadek T. CtsR, a novel regulator of stress and heat shock response, controls clp and molecular chaperone gene expression in Gram-positive bacteria. Mol Microbiol. 1999 Jan;31(1):117-31.

Derre I, Rapoport G, Msadek T. The CtsR regulator of stress response is active as a dimer and specifically degraded in vivo at $37^{\circ} \mathrm{C}$. Mol Microbiol. 2000 Oct;38(2):335-47.

Donegan NP, Thompson ET, Fu Z, Cheung AL. Proteolytic regulation of toxin-antitoxin systems by ClpPC in Staphylococcus aureus. J Bacteriol. 2010 Mar;192(5):1416-22.

Dubrac S, Boneca IG, Poupel O, Msadek T. New insights into the WalK/WalR (YycG/YycF) essential signal transduction pathway reveal a major role in controlling cell wall metabolism and biofilm formation in Staphylococcus aureus. J Bacteriol. 2007 Nov;189(22):8257-69.

Dursun F, Mohamoud HS, Karim N, Naeem M, Jelani M, Kırmızıbekmez H. A novel missense mutation in the CLPP gene causing Perrault syndrome type 3 in a Turkish family. J Clin Res Pediatr Endocrinol. 2016 Dec;8(4):472.
Elsholz AK, Gerth U, Hecker M. Regulation of CtsR activity in low GC, Gram+ bacteria. Adv Microb Physiol. 2010a;57:119-44.

Elsholz AK, Michalik S, Zühlke D, Hecker M, Gerth U. CtsR, the Gram-positive master regulator of protein quality control, feels the heat. EMBO J. 2010b Nov;29(21):3621-9.

Elsholz AKW, Birk MS, Charpentier E, Turgay K. Functional diversity of AAA+ protease complexes in Bacillus subtilis. Front Mol Biosci. 2017 Jul;4:44

Etter D, Schelin J, Schuppler M, Johler S. Staphylococcal enterotoxin C - an update on SEC variants, their structure and properties, and their role in foodborne intoxications. Toxins. 2020;12(9):584

Famulla K, Sass P, Malik I, Akopian T, Kandror $\mathrm{O}$, Alber M, et al. Acyldepsipeptide antibiotics kill mycobacteria by preventing the physiological functions of the ClpP1P2 protease. Mol Microbiol. 2016 Jul;101(2):194-209.

Farrand AJ, Reniere ML, Ingmer H, Frees D, Skaar EP. Regulation of host hemoglobin binding by the Staphylococcus aureus Clp proteolytic system. J Bacteriol. 2013;195(22): 5041-50.

Feng J, Michalik S, Varming AN, Andersen JH, Albrecht D, Jelsbak L, et al. Trapping and proteomic identification of cellular substrates of the ClpP protease in Staphylococcus aureus. J Proteome Res. 2013 Feb;12(2):547-58.

Fetzer C, Korotkov VS, Thänert R, Lee KM, Neuenschwander M, von Kries JP, et al. A chemical disruptor of the ClpX chaperone complex attenuates the virulence of multidrug-resistant Staphylococcus aureus. Angew Chem Int Ed Engl. 2017 Dec;56(49):15746-50.

Fischer F, Langer JD, Osiewacz HD. Identification of potential mitochondrial CLPXP protease interactors and substrates suggests its central role in energy metabolism. Sci Rep. 2015 Dec;5:18375.

Flynn JM, Levchenko I, Seidel M, Wickner SH, Sauer RT, Baker TA. Overlapping recognition determinants within the ssrA degradation tag allow modulation of proteolysis. Proc Natl Acad Sci USA. 2001 Sep;98(19):10584-9.

Frees D, Qazi SN, Hill PJ, Ingmer H. Alternative roles of ClpX and ClpP in Staphylococcus aureus stress tolerance and virulence. $\mathrm{Mol} \mathrm{Mi}$ crobiol. 2003 Jun;48(6):1565-78.

Frees D, Chastanet A, Qazi S, Sørensen K, Hill P, Msadek T, et al. Clp ATPases are required for stress tolerance, intracellular replication and biofilm formation in Staphylococcus aureus. Mol Microbiol. 2004 Dec;54(5):1445-62.

Frees D, Sørensen K, Ingmer H. Global virulence regulation in Staphylococcus aureus: pinpointing the roles of $\mathrm{ClpP}$ and $\mathrm{ClpX}$ in the sar/ agr regulatory network. Infect Immun. 2005 Dec;73(12):8100-8.

Frees D, Savijoki K, Varmanen P, Ingmer H. Clp ATPases and ClpP proteolytic complexes regulate vital biological processes in low GC, Gram-positive bacteria. Mol Microbiol. 2007 Mar;63(5):1285-95.
Frees D, Andersen JH, Hemmingsen L, Koskenniemi K, Bæk KT, Muhammed MK, et al. New insights into Staphylococcus aureus stress tolerance and virulence regulation from an analysis of the role of the ClpP protease in the strains Newman, COL, and SA564. J Proteome Res. 2012 Jan;11(1):95-108.

Gaillot O, Pellegrini E, Bregenholt S, Nair S, Berche P. The ClpP serine protease is essential for the intracellular parasitism and virulence of Listeria monocytogenes. Mol Microbiol. 2000 Mar;35(6):1286-94.

Gaillot O, Bregenholt S, Jaubert F, Di Santo JP, Berche P. Stress-induced ClpP serine protease of Listeria monocytogenes is essential for induction of listeriolysin O-dependent protective immunity. Infect Immun. 2001 Aug; 69(8):4938-43.

Garg SK, Kommineni S, Henslee L, Zhang Y, Zuber $\mathrm{P}$. The YjbH protein of Bacillus subtilis enhances ClpXP-catalyzed proteolysis of Spx. J Bacteriol. 2009 Feb;191(4):1268-77.

Gatsogiannis C, Balogh D, Merino F, Sieber SA, Raunser S. Cryo-EM structure of the ClpXP protein degradation machinery. Nat Struct Mol Biol. 2019 Oct;26(10):946-54.

Gerth U, Wipat A, Harwood CR, Carter N, Emmerson PT, Hecker M. Sequence and transcriptional analysis of clpX, a class-III heatshock gene of Bacillus subtilis. Gene. 1996 Nov; 181(1-2):77-83.

Gerth U, Kirstein J, Mostertz J, Waldminghaus T, Miethke M, Kock H, et al. Fine-tuning in regulation of Clp protein content in Bacillus subtilis. J Bacteriol. 2004 Jan;186(1):179-91.

Gispert S, Parganlija D, Klinkenberg M, Dröse S, Wittig I, Mittelbronn M, et al. Loss of mitochondrial peptidase Clpp leads to infertility, hearing loss plus growth retardation via accumulation of CLPX, mtDNA and inflammatory factors. Hum Mol Genet. 2013 Dec; 22(24):4871-87.

Glickman MS, Jacobs WR Jr. Microbial pathogenesis of Mycobacterium tuberculosis: dawn of a discipline. Cell. $2001 \mathrm{Feb}$;104(4):477-85.

Graves PR, Aponte-Collazo LJ, Fennell EMJ, Graves AC, Hale AE, Dicheva N, et al. Mitochondrial protease $\mathrm{ClpP}$ is a target for the anticancer compounds ONC201 and related analogues. ACS Chem Biol. 2019 May; 14(5): 1020-9.

Greene AW, Grenier K, Aguileta MA, Muise S, Farazifard R, Haque ME, et al. Mitochondrial processing peptidase regulates PINK1 processing, import and Parkin recruitment. EMBO Rep. 2012 Apr;13(4):378-85.

Grossman AD. Genetic networks controlling the initiation of sporulation and the development of genetic competence in Bacillus subtilis. Annu Rev Genet. 1995 Dec;29(1):477-508.

Guillon B, Bulteau AL, Wattenhofer-Donzé M, Schmucker S, Friguet B, Puccio H, et al. Frataxin deficiency causes upregulation of mitochondrial Lon and ClpP proteases and severe loss of mitochondrial Fe-S proteins. FEBS J. 2009 Feb;276(4):1036-47. 
Hall BM, Breidenstein EBM, de la Fuente-Núñez C, Reffuveille F, Mawla GD, Hancock REW, et al. Two Isoforms of Clp Peptidase in Pseudomonas aeruginosa control distinct aspects of cellular physiology. J Bacteriol. 2017 Feb; 199(3):e00568.

Hamoen LW, Eshuis H, Jongbloed J, Venema G, van Sinderen D. A small gene, designated coms, located within the coding region of the fourth amino acid-activation domain of srfA, is required for competence development in Bacillus subtilis. Mol Microbiol. 1995 Jan; 15(1):55-63.

Heidler J, Al-Furoukh N, Kukat C, Salwig I, Ingelmann ME, Seibel P, et al. Nitric oxide-associated protein 1 (NOA1) is necessary for oxygen-dependent regulation of mitochondrial respiratory complexes. J Biol Chem. 2011 Sep; 286(37):32086-93.

Ingvarsson $\mathrm{H}$, Maté MJ, Högbom M, Portnoï D, Benaroudj N, Alzari PM, et al. Insights into the inter-ring plasticity of caseinolytic proteases from the X-ray structure of Mycobacterium tuberculosis ClpP1. Acta Crystallogr D Biol Crystallogr. 2007 Feb;63(Pt 2):249-59.

Ishizawa J, Zarabi SF, Davis RE, Halgas O, Nii T, Jitkova Y, et al. Mitochondrial ClpP-mediated proteolysis induces selective cancer cell lethality. Cancer Cell. 2019 May;35(5):721-e9.

Jacquet R, Labauve AE, Akoolo L, Patel S, Alqarzaee AA, Wong Fok Lung T, et al. Dual gene expression analysis identifies factors associated with Staphylococcus aureus virulence in diabetic mice. Infect Immun. 2019 Mar;87(5): e00163-19.

Jelsbak L, Ingmer $\mathrm{H}$, Valihrach L, Cohn MT, Christiansen MH, Kallipolitis BH, et al. The chaperone ClpX stimulates expression of Staphylococcus aureus protein A by rot dependent and independent pathways. PLoS One. 2010 Sep;5(9):e12752.

Jensen C, Bæk KT, Gallay C, Thalsø-Madsen I, Xu $\mathrm{L}$, Jousselin A, et al. The ClpX chaperone controls autolytic splitting of Staphylococcus aureus daughter cells, but is bypassed by $\beta$-lactam antibiotics or inhibitors of WTA biosynthesis. PLoS Pathog. 2019a;15(9): e1008044.

Jensen C, Fosberg MJ, Thalsø-Madsen I, Bæk KT, Frees D. Staphylococcus aureus ClpX localizes at the division septum and impacts transcription of genes involved in cell division, T7-secretion, and SaPI5-excision. Sci Rep. 2019b;9(1):16456.

Kang SG, Ortega J, Singh SK, Wang N, Huang NN, Steven AC, et al. Functional proteolytic complexes of the human mitochondrial ATPdependent protease, hClpXP. J Biol Chem. 2002 Jun;277(23):21095-102.

Kang SG, Maurizi MR, Thompson M, Mueser T, Ahvazi B. Crystallography and mutagenesis point to an essential role for the $\mathrm{N}$-terminus of human mitochondrial ClpP. J Struct Biol. 2004 Dec;148(3):338-52.
Kim YI, Levchenko I, Fraczkowska K, Woodruff RV, Sauer RT, Baker TA. Molecular determinants of complex formation between Clp/ Hsp100 ATPases and the ClpP peptidase. Nat Struct Biol. 2001 Mar;8(3):230-3.

Kim GL, Akoolo L, Parker D. The ClpXP protease contributes to Staphylococcus aureus pneumonia. J Infect Dis. 2020 Sep;222(8):1400-4.

Kirsch VC, Fetzer C, Sieber SA. Global inventory of ClpP- and ClpX-regulated proteins in Staphylococcus aureus. J Proteome Res. 2021 Nov;20(1):867-79.

Kirstein J, Zühlke D, Gerth U, Turgay K, Hecker $\mathrm{M}$. A tyrosine kinase and its activator control the activity of the CtsR heat shock repressor in B. subtilis. EMBO J. 2005 Oct;24(19):343545.

Kirstein J, Dougan DA, Gerth U, Hecker M, Turgay $\mathrm{K}$. The tyrosine kinase $\mathrm{McsB}$ is a regulated adaptor protein for ClpCP. EMBO J. 2007 Apr;26(8):2061-70.

Kirstein J, Hoffmann A, Lilie H, Schmidt R, Rübsamen-Waigmann $\mathrm{H}$, Brötz-Oesterhelt $\mathrm{H}$, et al. The antibiotic ADEP reprogrammes ClpP, switching it from a regulated to an uncontrolled protease. EMBO Mol Med. 2009a Apr; 1(1):37-49.

Kirstein J, Molière N, Dougan DA, Turgay K. Adapting the machine: adaptor proteins for $\mathrm{Hsp} 100 / \mathrm{Clp}$ and AAA+ proteases. Nat Rev Microbiol. 2009b Aug;7(8):589-99.

Kock H, Gerth U, Hecker M. The ClpP peptidase is the major determinant of bulk protein turnover in Bacillus subtilis. J Bacteriol. 2004 Sep; 186(17):5856-64

Kolanczyk M, Pech M, Zemojtel T, Yamamoto H, Mikula I, Calvaruso MA, et al. NOA1 is an essential GTPase required for mitochondrial protein synthesis. Mol Biol Cell. 2011 Jan; 22(1):1-11.

Krüger E, Witt E, Ohlmeier S, Hanschke R, Hecker $\mathrm{M}$. The clp proteases of Bacillus subtilis are directly involved in degradation of misfolded proteins. J Bacteriol. 2000 Jun;182(11):325965.

Krüger E, Zühlke D, Witt E, Ludwig H, Hecker M. Clp-mediated proteolysis in Gram-positive bacteria is autoregulated by the stability of a repressor. EMBO J. 2001 Feb;20(4):852-63.

Kwon HY, Ogunniyi AD, Choi MH, Pyo SN, Rhee DK, Paton JC. The ClpP Protease of Streptococcus pneumoniae modulates virulence gene expression and protects against fatal pneumococcal challenge. Infect Immun. 2004 Oct;72(10):5646-53.

Larsson JT, Rogstam A, Von Wachenfeldt C. $\mathrm{YjbH}$ is a novel negative effector of the disulphide stress regulator, $\mathrm{Spx}$, in Bacillus subtilis. Mol Microbiol. 2007 Nov;66(3):669-84.

Lavey NP, Shadid T, Ballard JD, Duerfeldt AS. Clostridium difficile $\mathrm{ClpP}$ homologues are capable of uncoupled activity and exhibit different levels of susceptibility to acyldepsipeptide modulation. ACS Infect Dis. 2019 Jan;5(1):79-89.
Leodolter J, Warweg J, Weber-Ban E. The Mycobacterium tuberculosis ClpP1P2 protease interacts asymmetrically with its ATPase partners ClpX and ClpC1. PLoS One. 2015 May; 10(5):e0125345.

Lindsay JA, Ruzin A, Ross HF, Kurepina N, Novick RP. The gene for toxic shock toxin is carried by a family of mobile pathogenicity islands in Staphylococcus aureus. Mol Microbiol. 1998 Jul;29(2):527-43.

Liu J, Chien P. Structure and activation of a heteromeric protease complex. Proc Natl Acad Sci. 2014 Oct;111(43):15289-90.

Liu J, Zuber P. A molecular switch controlling competence and motility: competence regulatory factors ComS, MecA, and ComK control $\varsigma \mathrm{D}$ dependent gene expression in Bacillus subtilis. J Bacteriol. 1998 Aug;180(16):4243-51.

Lowy FD. Staphylococcus aureus infections. N Engl J Med. 1998 Aug;339(8):520-32.

Maglica Z, Striebel F, Weber-Ban E. An intrinsic degradation tag on the ClpA C-terminus regulates the balance of ClpAP complexes with different substrate specificity. J Mol Biol. 2008 Dec;384(2):503-11.

Malik IT, Brötz-Oesterhelt H. Conformational control of the bacterial Clp protease by natural product antibiotics. Nat Prod Rep. 2017 Jan;34(7):815-31.

Mckenney PT, Driks A, Eichenberger P. The Bacillus subtilis endospore: assembly and functions of the multilayered coat. Nat Rev Microbiol. 2013 Jan;11(1):33-44

Meyer AS, Baker TA. Proteolysis in the Escherichia coli heat shock response: a player at many levels. Curr Opin Microbiol. 2011 Apr; 14(2):194-9.

Michel A, Agerer F, Hauck CR, Herrmann M, Ullrich J, Hacker J, et al. Global regulatory impact of ClpP protease of Staphylococcus aureus on regulons involved in virulence, oxidative stress response, autolysis, and DNA repair. J Bacteriol. 2006 Aug; 188(16):5783-96.

Miethke M, Hecker M, Gerth U. Involvement of Bacillus subtilis ClpE in CtsR degradation and protein quality control. J Bacteriol. $2006 \mathrm{Jul}$; 188(13):4610-9.

Mirali S, Schimmer AD. The role of mitochondrial proteases in leukemic cells and leukemic stem cells. Stem Cells Transl Med. 2020 Aug; 9(12):1481-7.

Molière N, Hossmann J, Schäfer $\mathrm{H}$, Turgay $\mathrm{K}$. Role of Hsp100/Clp protease complexes in controlling the regulation of motility in Bacillus subtilis. Front Microbiol. 2016 Mar;7:315.

Msadek T, Dartois V, Kunst F, Herbaud ML, Denizot F, Rapoport G. ClpP of Bacillus subtilis is required for competence development, motility, degradative enzyme synthesis, growth at high temperature and sporulation. Mol Microbiol. 1998 Mar;27(5):899-914.

Mukherjee S, Kearns DB. The structure and regulation of flagella in Bacillus subtilis. Annu Rev Genet. 2014 Nov;48(1):319-40. 
Nagpal J, Paxman JJ, Zammit JE, Alhuwaider A, Truscott KN, Heras B, et al. Molecular and structural insights into an asymmetric proteolytic complex (ClpP1P2) from Mycobacterium smegmatis. Sci Rep. 2019 Dec;9(1): 18019.

Nair S, Frehel C, Nguyen L, Escuyer V, Berche P. ClpE, a novel member of the HSP100 family, is involved in cell division and virulence of Listeria monocytogenes. Mol Microbiol. 1999 Jan;31(1):185-96.

Nair S, Milohanic E, Berche P. ClpC ATPase is required for cell adhesion and invasion of Listeria monocytogenes. Infect Immun. 2000 Dec;68(12):7061-8.

Nakano MM, Zhu Y, Liu J, Reyes DY, Yoshikawa $\mathrm{H}$, Zuber P. Mutations conferring amino acid residue substitutions in the carboxy-terminal domain of RNA polymerase alpha can suppress clpX and clpP with respect to developmentally regulated transcription in Bacillus subtilis. Mol Microbiol. 2000 Aug;37(4):86984.

Nakano MM, Nakano S, Zuber P. Spx (YjbD), a negative effector of competence in Bacillus subtilis, enhances ClpC-MecA-ComK interaction. Mol Microbiol. 2002a;44(5):1341-9.

Nakano S, Zheng G, Nakano MM, Zuber P. Multiple pathways of Spx (YjbD) proteolysis in Bacillus subtilis. J Bacteriol. 2002b;184(13): 3664-70.

Nakano S, Nakano MM, Zhang Y, Leelakriangsak $\mathrm{M}$, Zuber P. A regulatory protein that interferes with activator-stimulated transcription in bacteria. Proc Natl Acad Sci USA. 2003 Apr;100(7):4233-8.

Olivares AO, Baker TA, Sauer RT. Mechanistic insights into bacterial AAA+ proteases and protein-remodelling machines. Nat Rev Microbiol. 2016 Jan;14(1):33-44.

Oliveira D, Borges A, Simões M. Staphylococcus aureus toxins and their molecular activity in infectious diseases. Toxins. 2018 Jun;10(6): 252.

Ollinger J, O'Malley T, Kesicki EA, Odingo J, Parish T. Validation of the essential ClpP protease in Mycobacterium tuberculosis as a novel drug target. J Bacteriol. 2012 Feb;194(3):663-8

Oshiro RT, Rajendren S, Hundley HA, Kearns DB. Robust stoichiometry of FliW-CsrA governs flagellin homeostasis and cytoplasmic organization in Bacillus subtilis. mBio. 2019 May;10(3):e00553.

Pan Q, Garsin DA, Losick R. Self-reinforcing activation of a cell-specific transcription factor by proteolysis of an anti- $\sigma$ factor in B. subtilis. Mol Cell. 2001 Oct;8(4):873-83.

Pan S, Malik IT, Thomy D, Henrichfreise B, Sass $\mathrm{P}$. The functional ClpXP protease of Chlamydia trachomatis requires distinct clpP genes from separate genetic loci. Sci Rep. 2019 Oct;9(1):14129.

Pavlova NN, Thompson CB. The emerging hallmarks of cancer metabolism. Cell Metab. 2016 Jan;23(1):27-47.
Pellegrino MW, Nargund AM, Haynes CM. Signaling the mitochondrial unfolded protein response. Biochim Biophys Acta. 2013 Feb; 1833(2):410-6.

Personne Y, Brown AC, Schuessler DL, Parish T. Mycobacterium tuberculosis ClpP proteases are co-transcribed but exhibit different substrate specificities. PLoS One. 2013 Jan;8(4): e60228.

Raju RM, Unnikrishnan M, Rubin DH, Krishnamoorthy V, Kandror O, Akopian TN, et al. Mycobacterium tuberculosis ClpP1 and ClpP2 function together in protein degradation and are required for viability in vitro and during infection. PLoS Pathog. 2012 Feb;8(2): e1002511.

Raju RM, Jedrychowski MP, Wei JR, Pinkham JT, Park AS, O'Brien K, et al. Post-translational regulation via Clp protease is critical for survival of Mycobacterium tuberculosis. PLoS Pathog. 2014 Mar;10(3):e1003994.

Rochat T, Nicolas P, Delumeau O, Rabatinová A, Korelusová J, Leduc A, et al. Genome-wide identification of genes directly regulated by the pleiotropic transcription factor Spx in $\mathrm{Ba}$ cillus subtilis. Nucleic Acids Res. 2012 Oct; 40(19):9571-83.

Rodgers AK, Wang J, Zhang Y, Holden A, Berryhill B, Budrys NM, et al. Association of tubal factor infertility with elevated antibodies to Chlamydia trachomatis caseinolytic protease P. Am J Obstet Gynecol. 2010 Nov;203(5): 494-e14.

Roels S, Driks A, Losick R. Characterization of spoIVA, a sporulation gene involved in coat morphogenesis in Bacillus subtilis. J Bacteriol. 1992 Jan;174(2):575-85.

Rojas-Tapias DF, Helmann JD. Identification of novel Spx regulatory pathways in Bacillus subtilis uncovers a close relationship between the CtsR and Spx regulons. J Bacteriol. 2019 Apr;201(13).

Runde S, Molière N, Heinz A, Maisonneuve E, Janczikowski A, Elsholz AK, et al. The role of thiol oxidative stress response in heat-induced protein aggregate formation during thermotolerance in Bacillus subtilis. Mol Microbiol. 2014 Mar;91(5):1036-52.

Schelin J, Cohn MT, Frisk B, Frees D. A functional ClpXP protease is required for induction of the accessory toxin genes, tst, sed, and sec. Toxins. 2020;12(9):553.

Schmitz KR, Sauer RT. Substrate delivery by the $\mathrm{AAA}+\mathrm{ClpX}$ and $\mathrm{ClpC} 1$ unfoldases activates the mycobacterial ClpP1P2 peptidase. Mol Microbiol. 2014;93(4):617-28.

Schmitz KR, Carney DW, Sello JK, Sauer RT. Crystal structure of Mycobacterium tuberculosis ClpP1P2 suggests a model for peptidase activation by AAA + partner binding and substrate delivery. Proc Natl Acad Sci USA. 2014; 111(43):E4587-95.

Schuster C, Bertram R. Toxin-antitoxin systems of Staphylococcus aureus. Toxins. 2016 May; $8(5): 140$.
Seiferling D, Szczepanowska K, Becker C, Senft K, Hermans S, Maiti P, et al. Loss of CLPP alleviates mitochondrial cardiomyopathy without affecting the mammalian UPRmt. EMBO Rep. 2016 May;17(7):953-64.

Seo JH, Rivadeneira DB, Caino MC, Chae YC Speicher DW, Tang HY, et al. The mitochondrial unfoldase-peptidase complex ClpXP controls bioenergetics stress and metastasis. PLoS Biol. 2016 Jul;14(7):e1002507.

Skaar EP, Schneewind O. Iron-regulated surface determinants (Isd) of Staphylococcus aureus: stealing iron from heme. Microbes Infect. 2004 Apr;6(4):390-7.

Spaulding AR, Salgado-Pabón W, Kohler PL, Horswill AR, Leung DY, Schlievert PM. Staphylococcal and streptococcal superantigen exotoxins. Clin Microbiol Rev. 2013 Jul; 26(3):422-47.

Stahlhut SG, Alqarzaee AA, Jensen C, Fisker NS, Pereira AR, Pinho MG, et al. The ClpXP protease is dispensable for degradation of unfolded proteins in Staphylococcus aureus. Sci Rep. 2017 Dec;7(1):11739.

Sun N, Youle RJ, Finkel T. The mitochondrial basis of aging. Mol Cell. 2016 Mar;61(5):654-66.

Szczepanowska K, Maiti P, Kukat A, Hofsetz E, Nolte H, Senft K, et al. CLPP coordinates mitoribosomal assembly through the regulation of ERAL1 levels. EMBO J. 2016 Dec;35(23): 2566-83.

Tan IS, Weiss CA, Popham DL, Ramamurthi KS, David, Ramamurthi S, et al. A quality-control mechanism removes unfit cells from a population of sporulating bacteria. Dev Cell. 2015 Sep;34(6):682-93.

Tanner AW, Carabetta VJ, Dubnau D. ClpC and MecA, components of a proteolytic machine, prevent Spo0A-P-dependent transcription without degradation. Mol Microbiol. 2018 Apr;108(2):178-86.

Thalsø-Madsen I, Torrubia FR, Xu L, Petersen A, Jensen C, Frees D. The Sle1 cell wall amidase is essential for $\beta$-lactam resistance in community-acquired methicillin-resistant Staphylococcus aureus USA300. Antimicrob Agents Chemother. 2019 Nov;64(1):e01931-19.

Trentini DB, Suskiewicz MJ, Heuck A, Kurzbauer R, Deszcz L, Mechtler K, et al. Arginine phosphorylation marks proteins for degradation by a Clp protease. Nature. 2016 Nov 3; 539(7627):48-53

Tsukahara K, Ogura M. Promoter selectivity of the Bacillus subtilis response regulator DegU, a positive regulator of the fla/che operon and sacB. BMC Microbiol. 2008 Jan;8(1):8.

Turgay K, Hamoen LW, Venema G, Dubnau D. Biochemical characterization of a molecular switch involving the heat shock protein $\mathrm{ClpC}$, which controls the activity of ComK, the competence transcription factor of Bacillus subtilis. Genes Dev. 1997 Jan;11(1):119-28.

Turgay K, Hahn J, Burghoorn J, Dubnau D. Competence in Bacillus subtilis is controlled by regulated proteolysis of a transcription factor. EMBO J. 1998 Nov;17(22):6730-8. 
Vahidi S, Ripstein ZA, Juravsky JB, Rennella E, Goldberg AL, Mittermaier AK, et al. An allosteric switch regulates Mycobacterium tuberculosis ClpP1P2 protease function as established by cryo-EM and methyl-TROSY NMR. Proc Natl Acad Sci USA. 2020 Mar;117(11): 5895-906.

Valera-Alberni M, Canto C. Mitochondrial stress management: a dynamic journey. Cell Stress. 2018 Oct;2(10):253-74.

Vestergaard M, Frees D, Ingmer H. Antibiotic resistance and the MRSA problem. Microbiol Spectr. 2019;7(2).

Voos W. Chaperone-protease networks in mitochondrial protein homeostasis. Biochim Biophys Acta. 2013 Feb;1833(2):388-99.

Wang C, Li M, Dong D, Wang J, Ren J, Otto M, et al. Role of ClpP in biofilm formation and virulence of Staphylococcus epidermidis. Microbes Infect. 2007 Sep;9(11):1376-83.

Wang Y, Hu M, Liu Q, Qin J, Dai Y, He L, et al. Role of the ESAT-6 secretion system in virulence of the emerging community-associated Staphylococcus aureus lineage ST398. Sci Rep. 2016 Apr;6(1):25163.

Weinandy F, Lorenz-Baath K, Korotkov VS, Böttcher T, Sethi S, Chakraborty T, et al. A $\beta$-lactone-based antivirulence drug amelio- rates Staphylococcus aureus skin infections in mice. ChemMedChem. 2014 Apr;9(4):710-3.

Wong KS, Houry WA. Chemical modulation of human mitochondrial ClpP: potential application in cancer therapeutics. ACS Chem Biol. 2019 Nov; 14(11):2349-60.

Wong KS, Mabanglo MF, Seraphim TV, Mollica A, Mao YQ, Rizzolo K, et al. Acyldepsipeptide analogs dysregulate human mitochondrial ClpP protease activity and cause apoptotic cell death. Cell Chem Biol. 2018 Aug;25(8): 1017-e9.

Wood NA, Blocker AM, Seleem MA, CondaSheridan M, Fisher DJ, Ouellette SP. The $\mathrm{ClpX}$ and ClpP2 orthologs of Chlamydia trachomatis perform discrete and essential functions in organism growth and development. mBio. 2020 Sep;11(5):e02016-20.

Yamada Y, Dick T. Mycobacterial caseinolytic protease gene regulator $\mathrm{ClgR}$ is a substrate of caseinolytic protease. mSphere. 2017 Mar; 2(2):e00338-16

Yu AY, Houry WA. ClpP: a distinctive family of cylindrical energy-dependent serine proteases. FEBS Lett. 2007 Jul;581(19):3749-57.

Zeiler E, Braun N, Böttcher T, Kastenmüller A, Weinkauf S, Sieber SA. Vibralactone as a tool to study the activity and structure of the
ClpP1P2 complex from Listeria monocytogenes. Angew Chem Int Ed Engl. 2011 Nov; 50(46):11001-4.

Zeiler E, List A, Alte F, Gersch M, Wachtel R, Poreba M, et al. Structural and functional insights into caseinolytic proteases reveal an unprecedented regulation principle of their catalytic triad. Proc Natl Acad Sci USA. 2013 Jul;110(28):11302-7.

Zhang Y, Zuber P. Requirement of the zinc-binding domain of ClpX for Spx proteolysis in Bacillus subtilis and effects of disulfide stress on ClpXP activity. J Bacteriol. 2007;189(21): 7669-80.

Zhang Y, Nakano S, Choi S-Y, Zuber P. Mutational analysis of the Bacillus subtilis RNA polymerase a C-terminal domain supports the interference model of Spx-dependent repression. J Bacteriol. 2006 Jun;188(12):4300-11.

Ziemski M, Leodolter J, Taylor G, Kerschenmeyer A, Weber-Ban E. Genome-wide interaction screen for Mycobacterium tuberculosis Clp$\mathrm{CP}$ protease reveals toxin-antitoxin systems as a major substrate class. FEBS J. 2021 Jan; 288(1):111-26.

Zong WX, Rabinowitz JD, White E. Mitochondria and cancer. Mol Cell. 2016 Mar;61(5): 667-76. 